\title{
Difunctionalization of Alkenes and Alkynes via Intermolecular Radical and Nucleophilic Additions
}

\author{
Hongjun Yao ${ }^{1,+}$, Wenfei $\mathrm{Hu}^{2,+}$ and Wei Zhang ${ }^{2, * \mathbb{C}}$ \\ 1 College of Biological Science and Technology, Beijing Forestry University, 35 Qinghua East Road, \\ Beijing 100083, China; hongjuny@bjfu.edu.cn \\ 2 Department of Chemistry, University of Massachusetts Boston, 100 Morrissey Boulevard, \\ Boston, MA 02125, USA; wenfei.hu@umb.edu \\ * Correspondence: wei2.zhang@umb.edu; Tel.: +1-617-287-6147 \\ + These authors contributed equally to this work.
}

Citation: Yao, H.; Hu, W.; Zhang, W. Difunctionalization of Alkenes and Alkynes via Intermolecular Radical and Nucleophilic Additions. Molecules 2021, 26, 105. https:// doi.org/10.3390/molecules26010105

Academic Editor: Derek J. McPhee Received: 7 December 2020 Accepted: 24 December 2020 Published: 28 December 2020

Publisher's Note: MDPI stays neutral with regard to jurisdictional clai$\mathrm{ms}$ in published maps and institutional affiliations.

Copyright: $(2020$ by the authors. Licensee MDPI, Basel, Switzerland. This article is an open access article distributed under the terms and conditions of the Creative Commons Attribution (CC BY) license (https:// creativecommons.org/licenses/by/ $4.0 /)$.

\begin{abstract}
Popular and readily available alkenes and alkynes are good substrates for the preparation of functionalized molecules through radical and/or ionic addition reactions. Difunctionalization is a topic of current interest due to its high efficiency, substrate versatility, and operational simplicity. Presented in this article are radical addition followed by oxidation and nucleophilic addition reactions for difunctionalization of alkenes or alkynes. The difunctionalization could be accomplished through 1,2-addition (vicinal) and 1,n-addition (distal or remote) if $\mathrm{H}$-atom or group-transfer is involved in the reaction process. A wide range of moieties, such as alkyl $(\mathrm{R})$, perfluoroalkyl $\left(\mathrm{R}_{\mathrm{f}}\right)$, aryl (Ar), hydroxy $(\mathrm{OH})$, alkoxy $(\mathrm{OR})$, acetatic $\left(\mathrm{O}_{2} \mathrm{CR}\right)$, halogenic $(\mathrm{X})$, amino $\left(\mathrm{NR}_{2}\right)$, azido $\left(\mathrm{N}_{3}\right)$, cyano $(\mathrm{CN})$, as well as sulfur- and phosphorous-containing groups can be incorporated through the difunctionalization reactions. Radicals generated from peroxides or single electron transfer (SET) agents, under photoredox or electrochemical reactions are employed for the reactions.
\end{abstract}

Keywords: difunctionalization; radical; nucleophilic; alkene; alkyne; photoredox; single electron transfer; vicinal-difunctionalization; distal-difunctionalization; photoredox; electrochemical reaction

\section{Introduction}

Radical-based reactions such as homolytic bond cleavage, single electron transfer, atom or group transfer, radical addition, and radical coupling processes are important organic transformations. In addition to the success of radical polymerization for making synthetic polymers, organic radical reactions have also become an increasing important tools for preparation of small molecules through radical addition, cyclization, cascade bond formation, $\mathrm{H}$-atom and group-transfer, and radical-radical coupling reactions [1,2]. Active researches on direct C-H bond functionalization [3], remote (distal) functionalization [4], Smile-type ipso-group rearrangement [5], cascade reaction [6], photoredox catalysis [7], and electrochemical reaction [8] have significantly fueled the chemistry of synthetic radicals.

Radical difunctionalization of unsaturated carbon-carbon bonds is a topic of current interest due to its high synthetic efficiency, substrate versatility, and operational simplicity [9-17]. Presented in this article are one-pot radical addition, oxidation and nucleophilic addition reactions to incorporate two functional groups to alkenes or alkynes. The difunctionalization could be accomplished through direct 1,2-addition (Scheme 1I-A), 1,2-functionalization involving Smiles-type ispo aryl group migration (Scheme 1I-B) [4,5], or 1,n-functionalization involving H-atom transfer (Scheme 1I-C) [4-6]. The majority examples in this paper are direct 1,2-additions. Only a few cases are related to 1,2-functionalization through Smiles-type rearrangement and distal difunctionalizations through 1,5-H-atom transfer. A wide range of moieties, such as alkyl $(\mathrm{R})$, perfluoroalkyl $\left(\mathrm{R}_{\mathrm{f}}\right)$, aryl (Ar), hydroxy $(\mathrm{OH})$, alkoxy $(\mathrm{OR})$, acetatic $\left(\mathrm{O}_{2} \mathrm{CRRCO}_{2}\right)$, halogenic $(\mathrm{X})$, amino $\left(\mathrm{NR}^{2}\right)$, azido $\left(\mathrm{N}_{3}\right)$, cyano $(\mathrm{CN})$, as well as sulfur- and phosphorous-containing groups can be incorporated through 
difunctionalization reactions. Radical reactions initiated with peroxides (such as dicumyl peroxide (DCP), di-t-butyl peroxide (DTBP), and $t$-butyl hydroperoxide (TBHP)) [18], promoted by single electron transfer agents (such as hypervalent iodine reagents (HIRs), ceric ammonium nitrate $(\mathrm{CAN}), \mathrm{Mn}(\mathrm{OAc})_{2}$, and $\left.\mathrm{Na}_{2} \mathrm{~S}_{2} \mathrm{O}_{5}\right)$, under photoredox catalysis (such as $\mathrm{Ru}(\mathrm{bpy})_{3} \mathrm{Cl}_{2}$ and $\operatorname{Ir}(\mathrm{ppy})_{3}$ ) [7], and by electrochemical reactions [8] are included. In the radical-initiated difunctionalization reactions, the catalysts are critical for oxidative SET to convert the intermediate radicals to carbocations for the sequential reaction with nucleophiles.

This paper covers difunctionalization reactions which are initiated with a radical addition and ended with a nucleophilic addition. It is organized based on the nature of initial radicals including $\mathrm{C}-_{-} \mathrm{N}-, \mathrm{O}-$, $\mathrm{P}-$-, $\mathrm{S}$-centered and perfluoroalkyl $\left(\mathrm{R}_{\mathrm{f}}\right)$ radicals. Reactions involving electrophilic addition (Scheme 1II-A), radical-radical coupling such as atom transfer radical addition (ATRA) [19] or metal-catalyzed coupling (Scheme 1II-B) [20], radical cyclization for ring formation (Scheme 1II-C) [21], and cascade reactions [6] involving more than two steps of radical additions are not included in this paper. Those type of reactions might be the topics of our future review articles.

I) Reactions covered in this paper

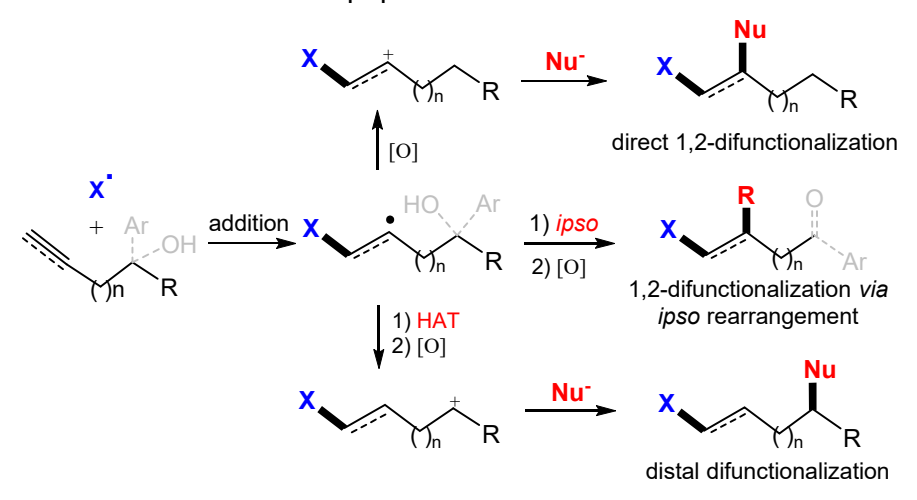

II) Reactions not covered in this paper

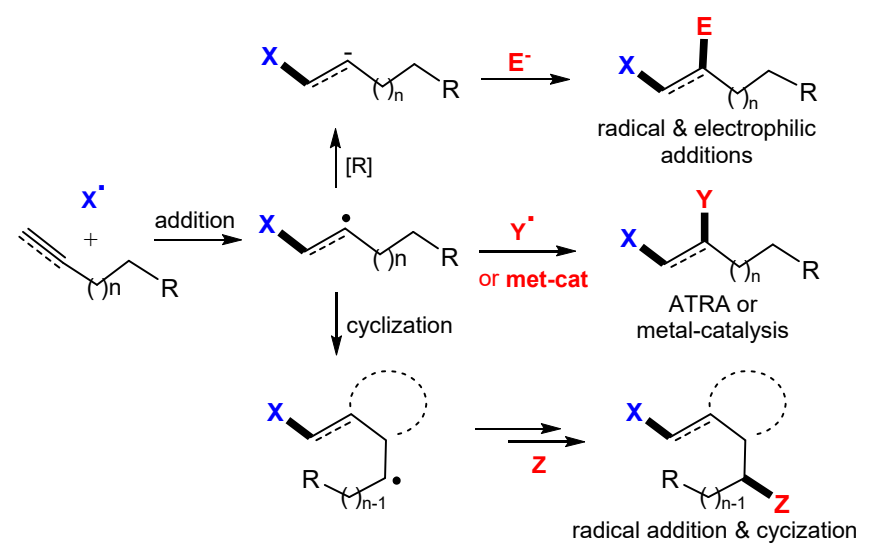

(A)

Scheme 1. Radical-initiated difunctionalization reactions. (I-A), direct 1,2-difuctionalization. (I-B), 1,2-difuctionalization involving ipso-reaction process. (I-C), distal difunctionalization. (II-A), 1,2difuctionalization involving electrophilic addition. (II-B), atom-transfer addition with or without metal catalysis. (II-C), reaction involving cyclization.

\section{Carbon Radical-Initiated Reactions}

Presented in this section are carbon radical-initiated reactions and C-, $\mathrm{O}-$, and Ncontaining moieties are served as the nucleophiles for the difunctionalizations. The initial carbon radicals could be generated by metal-containing SET agents, using peroxides, or under photoredox conditions. Most alkenes used for difunctionalization are styrene derivatives. Because addition of initial radicals to styrenes to form stabilized benzylic 
radicals is regioselective which could be readily oxidized to benzylic cations for nucleophilic additions.

The Du and Wang labs developed a highly efficient Re-catalyzed oxyalkylation of alkenes using hypervalent iodine reagents (HIRs) as a dual functionalization agent to provide alkyl radicals and carboxylic anions for additions (Scheme 2) [22]. In the reaction process, cationic species 2 generated through a heterolytic cleavage of an I-O bond from HIR are reduced by $\operatorname{Re}^{\mathrm{I}}$ to afford radical iodine intermediates 3 . Homolytic breaking of the remaining I-O bond gives acyloxy radicals which undergo decarboxylation to generate alkyl radicals. Addition of alkyl radicals to alkene followed by oxidation with $\mathrm{Re}^{\mathrm{I}}$ give cations 4 . Finally, nucleophilic attack of 4 by carboxylic anions gives difunctionalized products 1 .

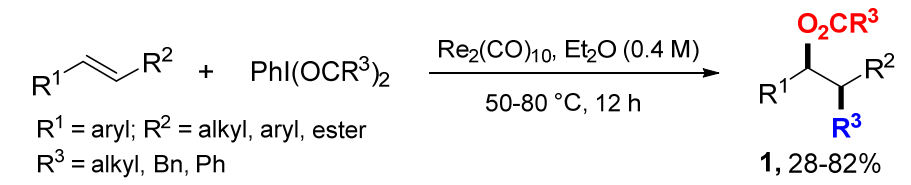

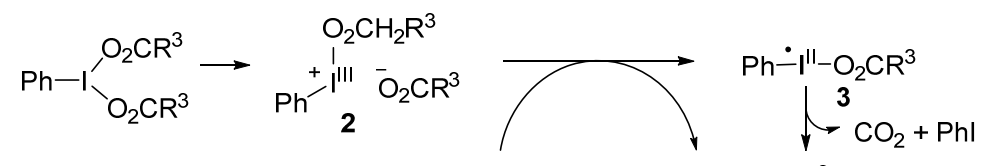

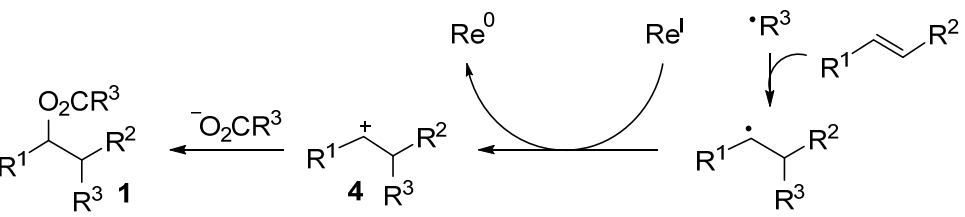

Scheme 2. Re-catalyzed difunctionalization with HIRs.

The Li group reported Ag-mediated 1,2-alkylarylation of styrenes with $\alpha$-carbonyl alkyl bromides and indoles (Scheme 3) [23]. The Fe-salt is used as Lewis acid to stabilize the radicals.The initial alkyl radicals 6 produced from $\alpha$-carbonyl alkyl bromides in the presence of 2 equiv of $\mathrm{Ag}^{\mathrm{I}}$ salt add to styrenes to afford radicals 6 which are oxidized by $\mathrm{Ag}$ II to cations and then undergo the Friedel-Crafts reaction with indoles to give products 5 .
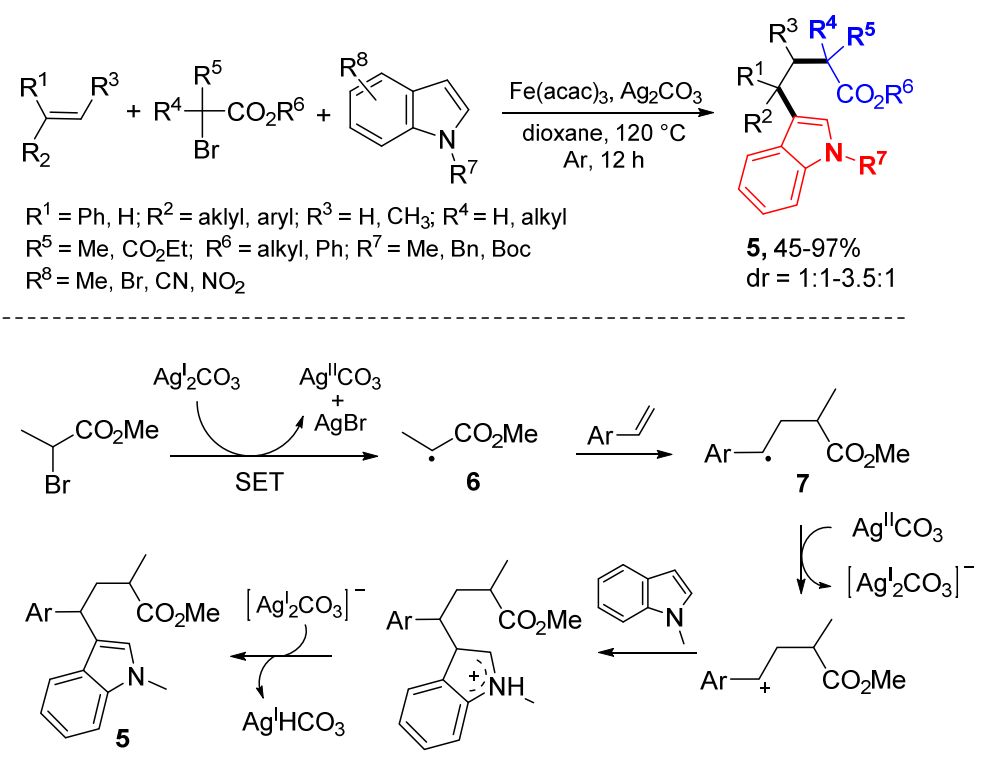

Scheme 3. Ag-catalyzed 1,2-alkylarylation of styrenes. 
The Bao group reported Fe-catalyzed decarboxylative alkyl etherification of vinylarenes with aliphatic acids and alcohols (Scheme 4) [24]. Primary, secondary and tertiary aliphatic acids could be used as alkylating reagents, and primary and secondary alcohols as nucleophiles. Alkyl radicals generated from the reaction of $\mathrm{Fe}^{\mathrm{II}} \mathrm{X}_{2}$ complex with peresters add to styrenes to form benzylic radicals which are oxidized to carbocations by $\mathrm{Fe}^{\mathrm{III}}$ species and then undergo nucleophilic reaction to give products 8 .
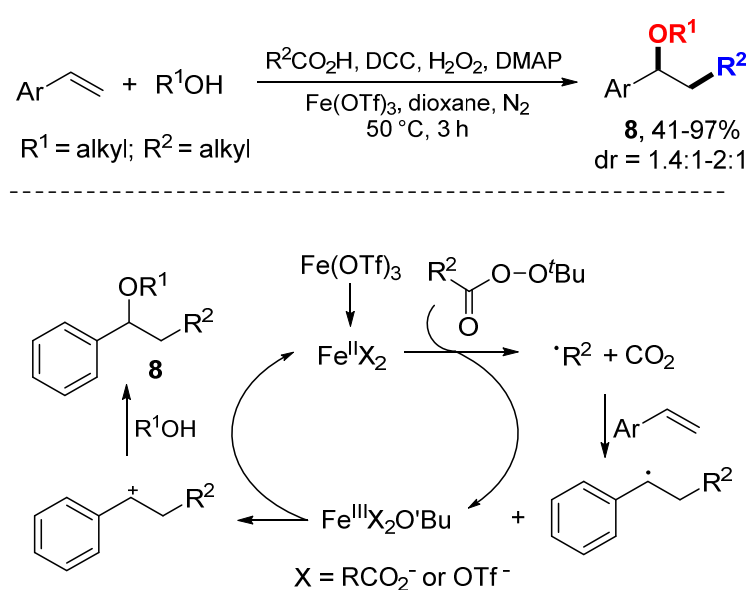

Scheme 4. Fe-catalyzed alkyl etherification with aliphatic acids.

The Nishikat group reported a Cu-catalyzed reaction for difunctionalizing styrenes using $\alpha$-bromocarbonyl compounds as a source for tertiary alkyl radicals and alcohols as nucleophiles (Scheme 5) [25]. Single electron transfer (SET) of $\mathrm{Cu}^{\mathrm{I}}$ induces the formation of radicals $\mathbf{1 0}$ from $\alpha$-bromocarbonyl compounds. The addition of radicals to styrenes followed by oxidation with $\mathrm{Cu}^{\mathrm{II}}$ to benzylic cations and trapping with alcohol nucleophiles give products 9. It was found that Lewis acids (LA), such as $\mathrm{Zn}(\mathrm{OTf})_{2}$, could accelerate the nucleophilic addition of alcohols.

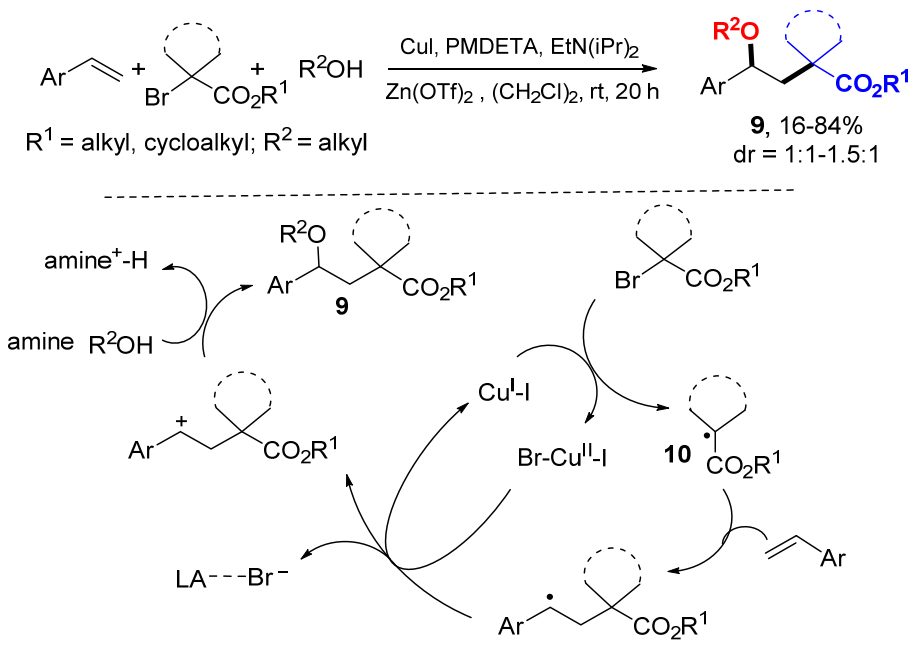

Scheme 5. Cu-catalyzed difunctionalization of styrenes.

The Yang group reported Fe-catalyzed decarbonylative alkylazidation of styrenes with aliphatic aldehydes and trimethylsilyl azide (Scheme 6) [26]. Using $(t-\mathrm{BuO})_{2}(\mathrm{DTBP})$ as an oxidant and radical initiator, a series of aliphatic aldehydes were converted to primary, secondary and tertiary alkyl radicals to react with styrenes followed by nucleophilic azidation with $\mathrm{TMSN}_{3}$. In the presence of the Fe catalyst, $t$-butoxy radical formed through the homolytic cleavage of DTBP abstracts the hydrogen from aldehydes followed by decarbonylation to give alkyl radicals which then react with styrenes to from benzylic 
radicals. Formation of cations by oxidization followed with the reaction of $\mathrm{TMSN}_{3}$ give products 11. The authors also proposed another mechanism of radical coupling in the formation of products.

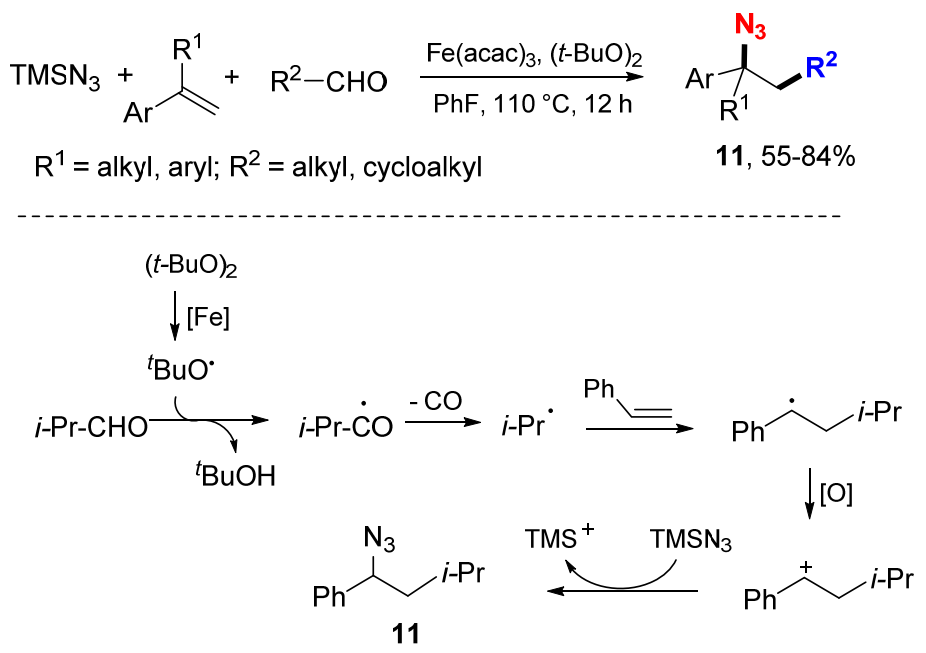

Scheme 6. Fe-catalyzed alkylazidation of styrenes.

The Zhu group reported a Cu-catalyzed methylalkoxy difunctionalization of alkenes using dicumyl peroxide (DCP) as a methyl source and alcohols as nucleophiles (Scheme 7) [27]. The $t$-alkoxy radical generated from the cleavage of DCP undergoes $\beta$-scission to release the methyl radical. It is captured by alkenes to produce benzyl radicals $\mathbf{1 3}$ which are oxidized to carbeniums by $\mathrm{Cu}^{\mathrm{II}}$ and then react with alcohols to furnish the methylated ether products 12.

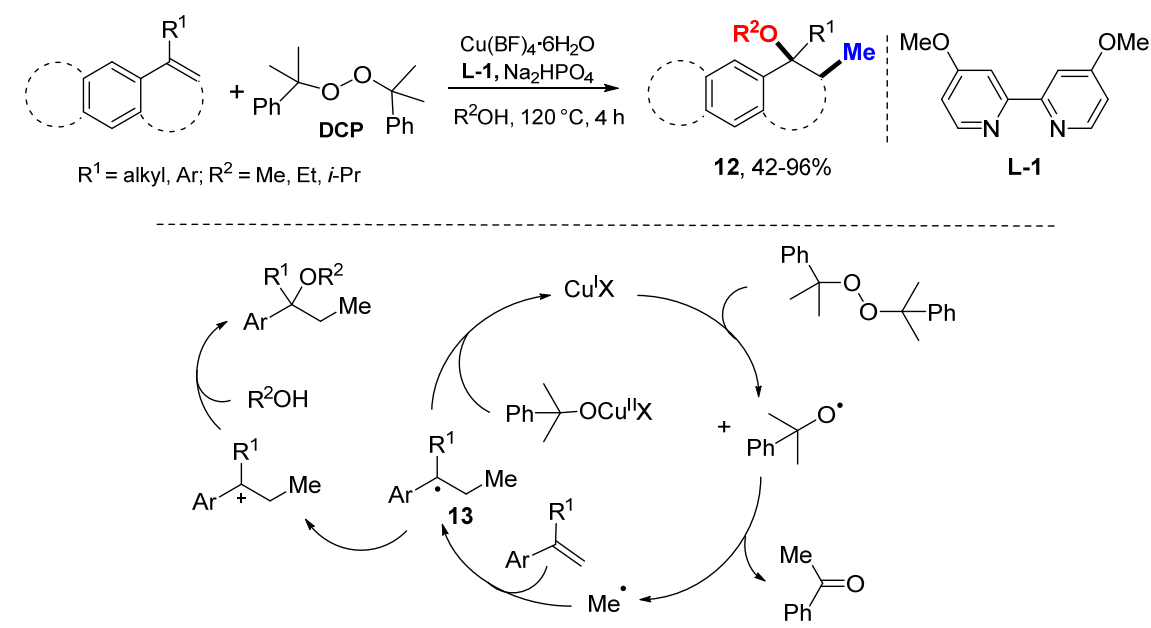

Scheme 7. Cu-catalyzed methylalkoxyl difunctionalization of styrenes.

The Klussmann group reported a reaction for difunctionalization of styrenes with carbon radicals derived from thioxanthene/xanthene and thiophenols followed by nucleophilic addition of acetyl nitrile or alcohols (Scheme 8) [28]. The combination of benzoyl peroxide (BPO) with HPF6 is a key factor of this reaction. The benzoyloxyl radical generated from $\mathrm{BPO}$ abstracts $\mathrm{H}$-atom from thioxanthene to give a new radical which then adds to styrenes to form the benzylic cations $\mathbf{1 5}$ after oxidation with BPO in the presence of HPF6. Acetonitrile or alcohols react with the carbocations in the fashion of a Ritter reaction to generate products $14 \mathbf{a}$ or $\mathbf{1 4 b}$. 


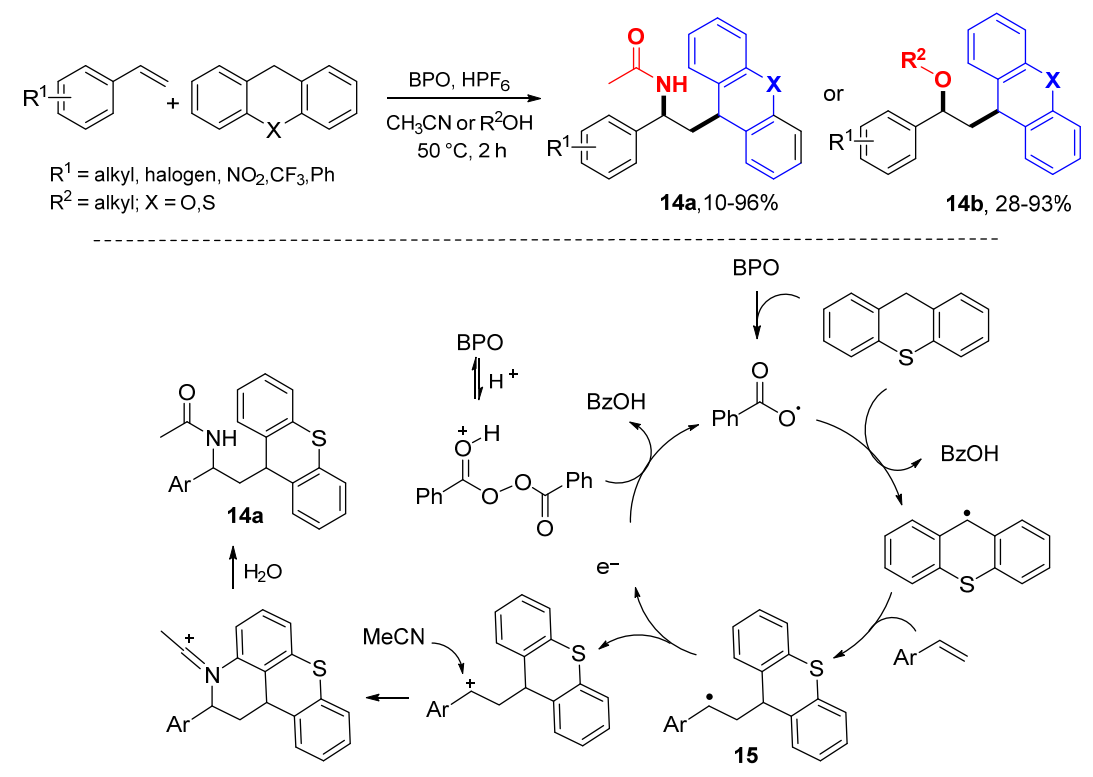

Scheme 8. Nitro- and oxyalkylation of styrenes.

The Greaney group reported photoredox catalytic arylalkoxy or alkylamino difunctionalization of styrenes using $\mathrm{Zn}(\mathrm{OAc})_{2}$ to enhance the reaction process (Scheme 9) [29]. Aryl radicals generated from the reduction of $\mathrm{Ar}_{2} \mathrm{IBF}_{4}$ under the catalysis of $\mathrm{Ir}^{\mathrm{III}}$ add to styrenes to form benzylic radicals which are oxidized with $\operatorname{Ir}^{\mathrm{IV}}$ to cations. Addition of nucleophiles (alcohols, $\mathrm{H}_{2} \mathrm{O}$ or nitriles) afford difunctionalized products 16.

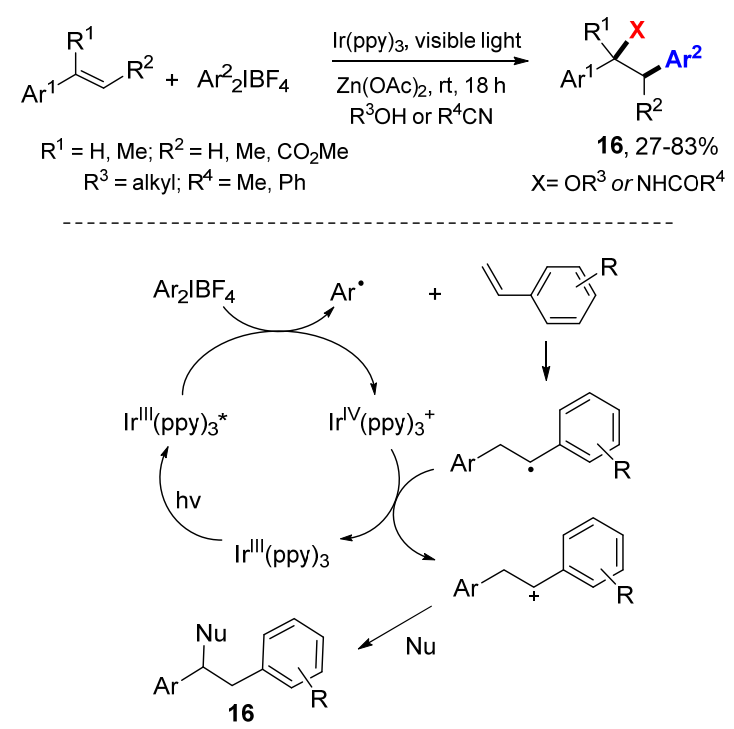

Scheme 9. Aryl/alkyloxy or alkylamino difunctionalization of styrenes. * excited catalyst.

The Glorius lab developed a method for alkyloxylation of styrenes through photo electron transfer-initiated reaction of $\mathrm{N}$-(acyloxy)phthalimides (Scheme 10) [30]. Water and alcohols act as nucleophiles and hydrogen-bond donors to afford alcohol and ether products 17. The reactions involve SET of light-emitting diode (LED) excited $\left[\operatorname{Ir}(\mathrm{ppy})_{2}(\mathrm{dtbbpy})\right]^{+}$ with the hydrogen bond complexes 18 to give radical anions 19, which then undergo N-O bond cleavage followed by decarboxylation to afford alkyl radicals. Radical addition to olefin followed by oxidation with $\left[\operatorname{Ir}(\mathrm{ppy})_{2}(\mathrm{dtbbpy})\right]^{2+}$ afford cations which are trapped by nucleophiles to afford alkyloxylated products $\mathbf{1 7}$. 


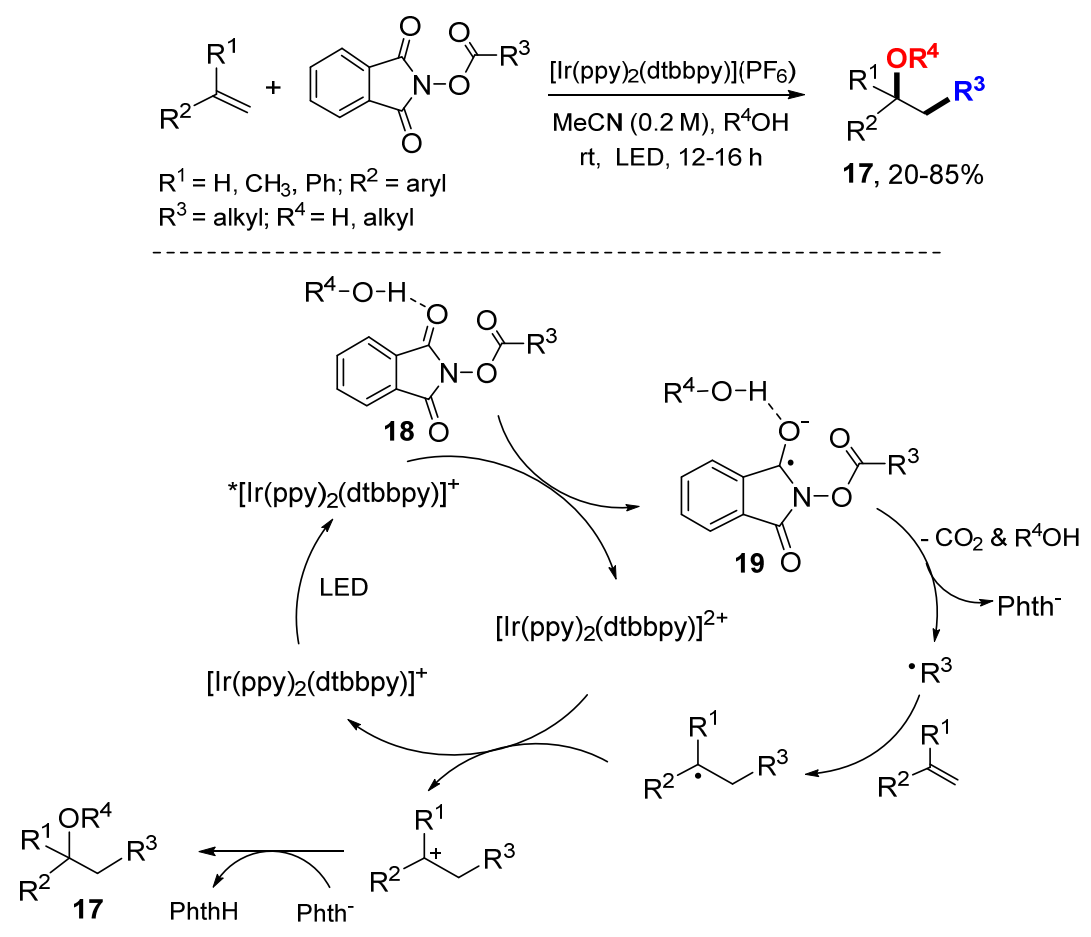

Scheme 10. Visible-light-mediated alkyloxylation. * excited catalyst.

The $\mathrm{Li}$ lab reported an alkylaryl reaction of styrenes with $\alpha$-carbonyl alkyl bromides and $N, N$-disubstituted anilines under photoredox catalysis of $\mathrm{Ru}(\mathrm{bpy})_{3} \mathrm{Cl}_{2}$ in the presence of a $\mathrm{Cu}$ salt (Scheme 11) [31]. The light excited catalyst $\mathrm{Ru}(\mathrm{bpy})_{3}{ }^{2+}$ transfers single electron to ethyl 2-bromo-2-methylpropanoates to form alkyl radicals 21 which add to styrenes, oxidized by $\mathrm{Ru}(\mathrm{bpy})_{3}{ }^{3+}$ to cationic intermediates 22 , followed by the Friedel-Crafts reaction to give products 20.

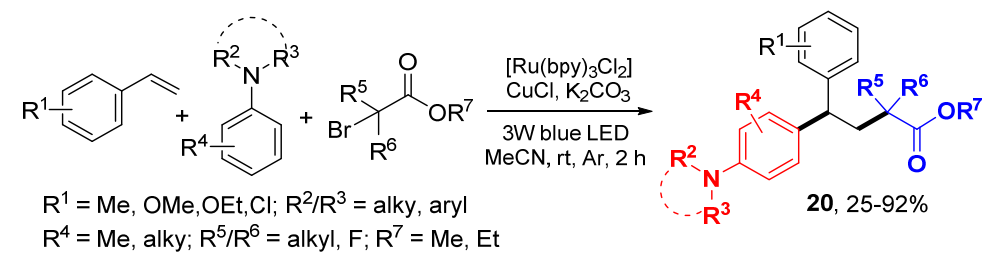<smiles>[R20]c1ccc(OCC)cc1</smiles><smiles>[3H]CC(C)(C)C(C)(CC(c1ccc(C(CC(C)(C)C(=O)OCC)c2ccc(OC)cc2)cc1)C1C=CC(=[N+](C)C)C=C1)C(=O)OCC</smiles>

Scheme 11. Alkyl \& aryl difunctionalization of styrenes. * excited catalyst. 
The Klussmann group reported an Ir-promoted photoredox reaction of styrenes with aldehydes and $N$-alkylindoles (Scheme 12) [32]. Both aryl and aliphatic aldehydes could be used for the generation of acyl radicals. $t$-Butoxyl radical generated through the SET of photo-excited $\mathrm{Ir}^{\mathrm{III}}$ and $t \mathrm{BuOOBz}$ (TBPB) react with aldehydes to form acyl radicals which add to styrenes to from 24 which then oxidized with $\mathrm{Ir}^{\mathrm{IV}}$ to carbocations. The Friedel-Crafts reaction of carbocations with $N$-alkylindoles give final products 23 .
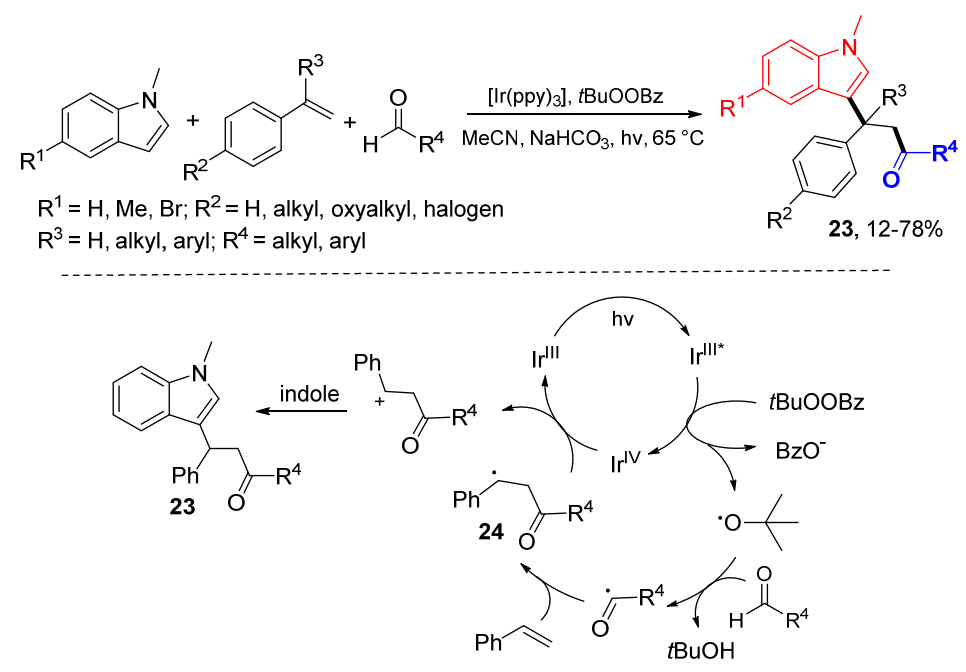

Scheme 12. Acyl radical and $N$-alkylindole difunctionalization of styrenes. * excited catalyst.

\section{Nitrogen Radical-Initiated Reactions}

Presented in this section are azido radical $\left(\mathrm{N}_{3} \cdot\right)$-initiated difunctionalization reactions. The azido radical can be generated from the Zhdankin's reagent or TMSN 3 [33].

The Zhu group developed a transition-metal-free reaction for azidocyanation of alkenes involving 1,4- or 1,5-cyano migration (Scheme 13) [34]. An azido radical generated from the reaction of $\mathrm{TMSN}_{3}$ with $\mathrm{PhI}(\mathrm{OAc})_{2}$ adds to cyanohydrin alkenes. The resulted radicals undergo 1,4-cyano group migration $(n=1)$ through a cyclization and ring-opening process to give more stable hydroxyalkyl radicals 25 . Oxidation of the radicals with $\mathrm{PhI}(\mathrm{OAc})_{2}$ (PIDA) followed by deprotonation affords the desired product. The Zhu group applied this strategy for the reaction of $\gamma$-hydroxyalkenes in the synthesis of 1,2-difuctionalized compounds 26 involving the 1,4-heteroaryl group migration (Scheme 14) [35].
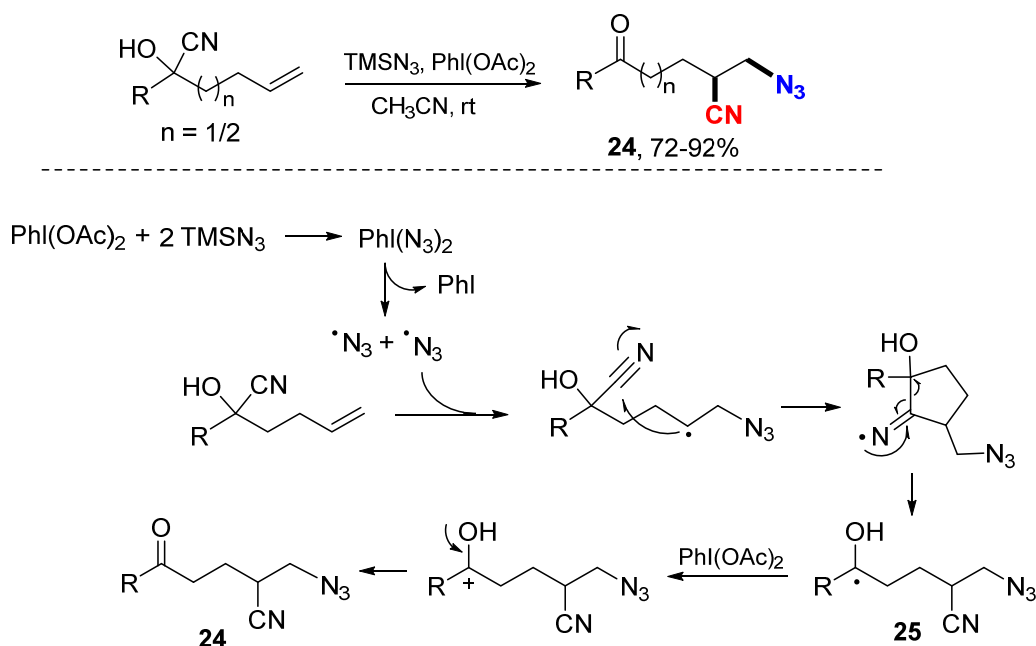

Scheme 13. Azidocyanation of olefins involving cyano migration. 

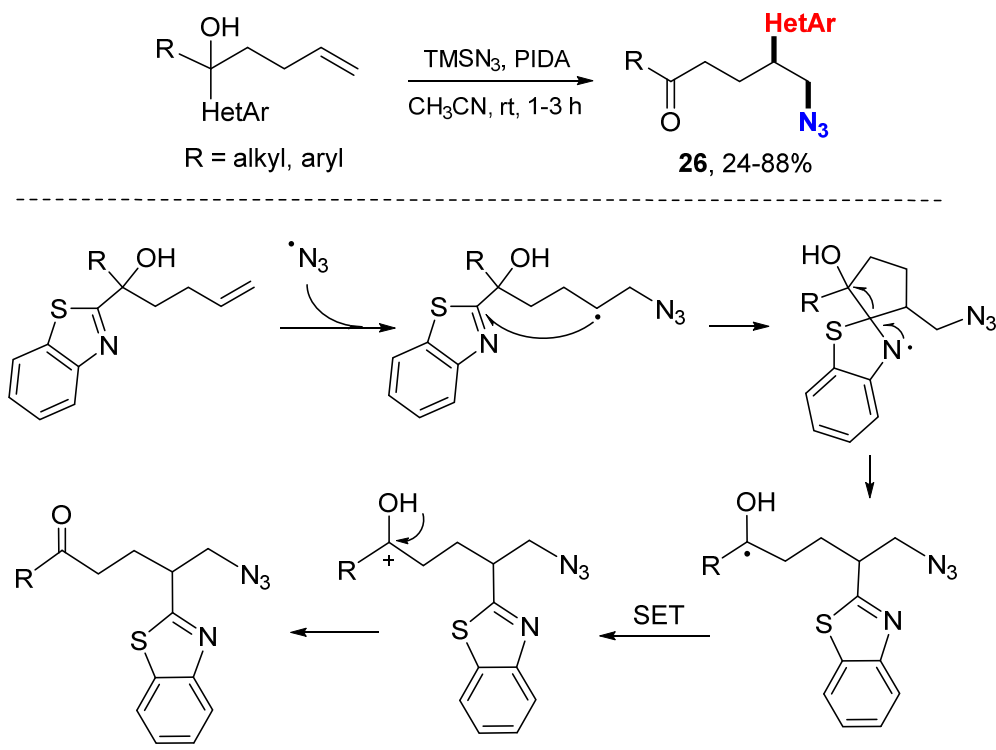

Scheme 14. Azidoheteroarylation involving heteroaryl migration.

The Tang group reported an azidotrifluoromethoxylation of styrenes with Zhdankin's reagent and trifluoromethyl arylsulfonate (TFMS) (Scheme 15) [36]. The reactions were conducted under photoredox conditions using $\mathrm{Ru}$ and $\mathrm{Ag}$ as dual catalysts. $\left[\mathrm{Ru}(\mathrm{bpy})_{3}\right]^{2+}$ Promoted formation of an azido radical from Zhdankin's reagent adds to styrenes followed by oxidation to benzyl carbocations 28 and Ag-catalyzed trifluoromethoxylation give desired products 27 . The reaction tolerates a wide range of functional groups and is applicable to late-stage azidotrifluoromethoxylation of more complicated molecules.
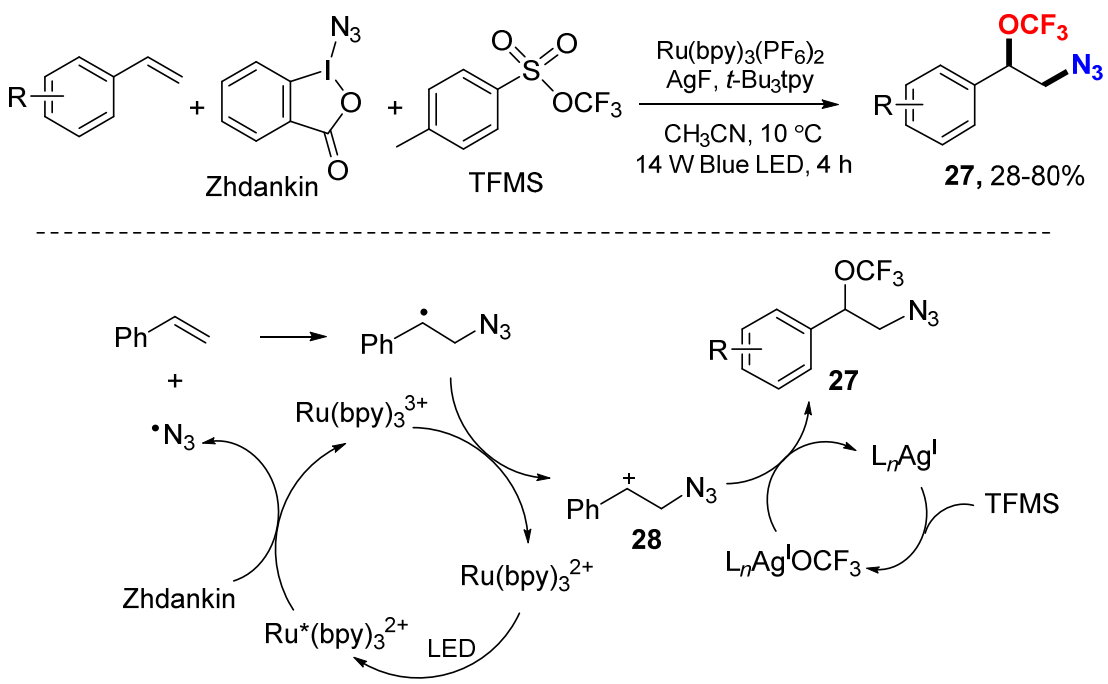

Scheme 15. Ag/Ru-catalyzed azidotrifluoromethoxylation of styrenes. * excited catalyst.

Zhu and coworkers developed a photoredox reaction for 1,2-difuctionalization of 1,3-dienes with $\mathrm{N}$-aminopyridinium salts and TMSNCS (Scheme 16) [37]. Under the photocatalysis of $f a c-\operatorname{Ir}(\mathrm{ppy})_{3}$, amino radicals derived from the $\mathrm{N}$-aminopyridinium salts add to 1,3-dienes to form allylic radicals $\mathbf{3 0}$ which are oxidized to cations followed by a reaction with TMSNCS to give difunctionalized products 29. 


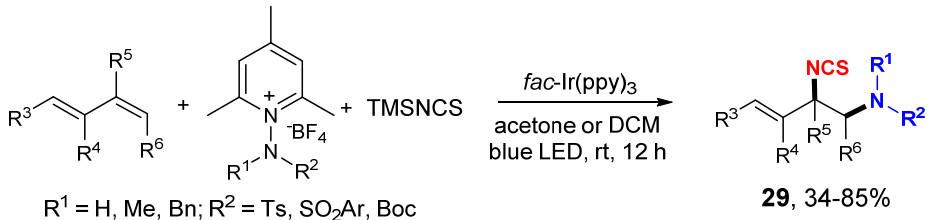

$\mathrm{R}^{1}=\mathrm{H}, \mathrm{Me}, \mathrm{Bn} ; \mathrm{R}^{2}=\mathrm{Ts}, \mathrm{SO}_{2} \mathrm{Ar}, \mathrm{Boc}$

29, $34-85 \%$

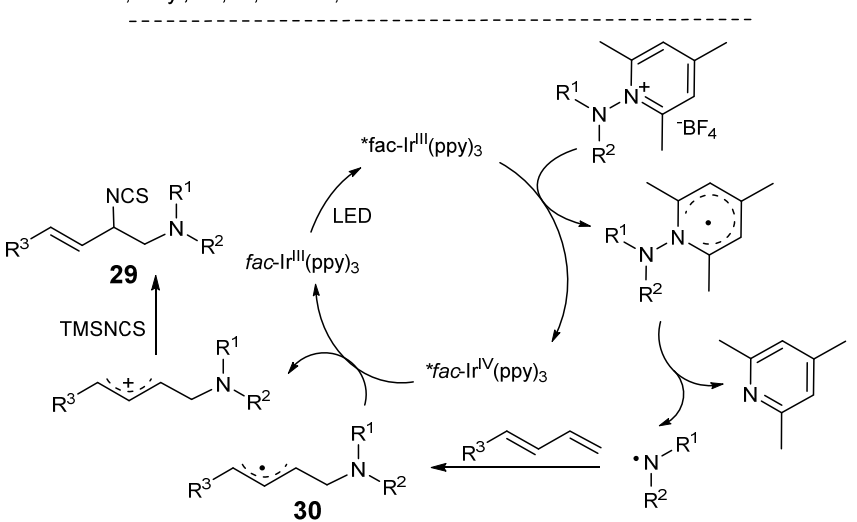

Scheme 16. Difunctionalization of 1,3-dienes. * excited catalyst.

\section{Oxygen Radical-Initiated Reactions}

Difunctionalization reactions of unsaturated carbon double bonds initiated with the addition of oxygen radicals are limited in literature. The oxygen radicals described in this section are alkoxy, acyl and phthalimide $\mathrm{N}$-oxyl (PINO) radicals which are generated from the homolytic cleavage of $\mathrm{N}-\mathrm{O}$ or $\mathrm{O}-\mathrm{H}$ bonds.

The Zhu group developed $t$-butyl hydroperoxide (TBHP)-induced synthesis of $\alpha$-hydroxy esters 31a or $\alpha$-hydroxy amines 31b by the reaction of alkenes with carboxylic acids or amines in the presence of $\mathrm{H}_{2} \mathrm{O}$ and using TBHP (70\% in water) as an oxidant (Scheme 17) [38]. $t$-Butoxyl and $t$-butylperoxy radicals generated from TBHP induce the formation of carboxyl radicals which add to styrenes followed by oxidation to benzyl cations and trapped with $\mathrm{H}_{2} \mathrm{O}$ to give products 31a.

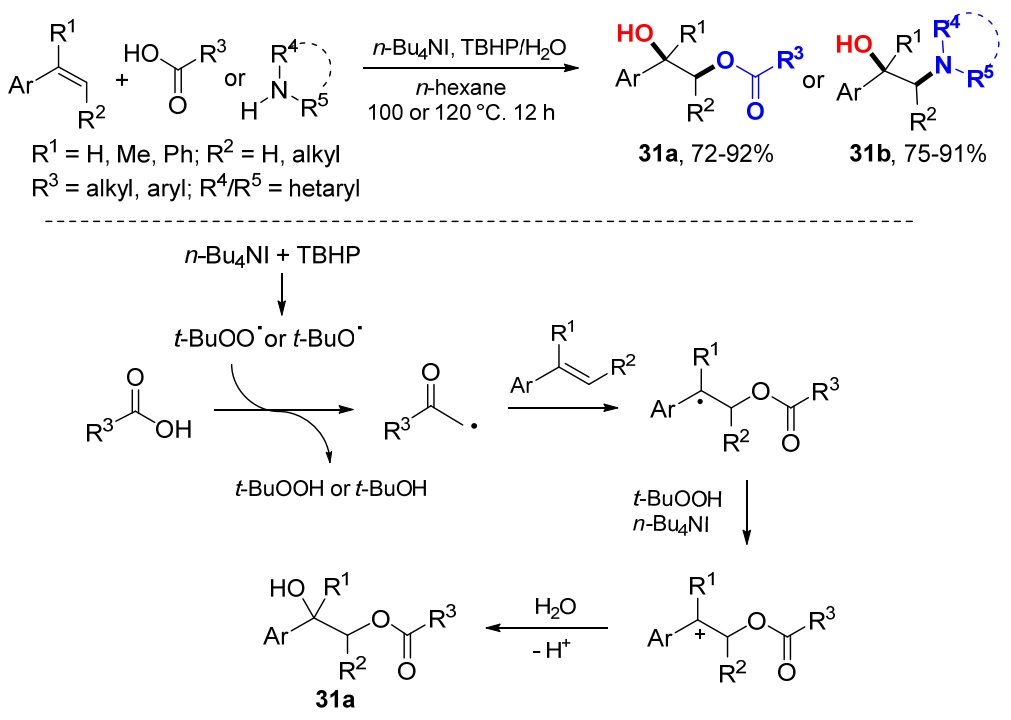

Scheme 17. $n$-Bu $\mathrm{Bu}_{4} \mathrm{NI}$-catalyzed difuctionalization of alkenes.

The Xia group reported a method for oxyazidation of alkenes with $\mathrm{TMSN}_{3}$ and $\mathrm{N}$-hydroxyphthalimide (NHPI) for making 2-azido-2-(phenylethoxy)isoindolinone compounds 32 (Scheme 18) [39]. The oxygen-centered PINO radical generated from NHPI 
reacts with styrenes followed by oxidation to carbocations with $\mathrm{PhI}(\mathrm{OAc})_{2}$ to from cations 33 which react with $\mathrm{N}_{3}{ }^{-}$to give oxyazidation products 32 .
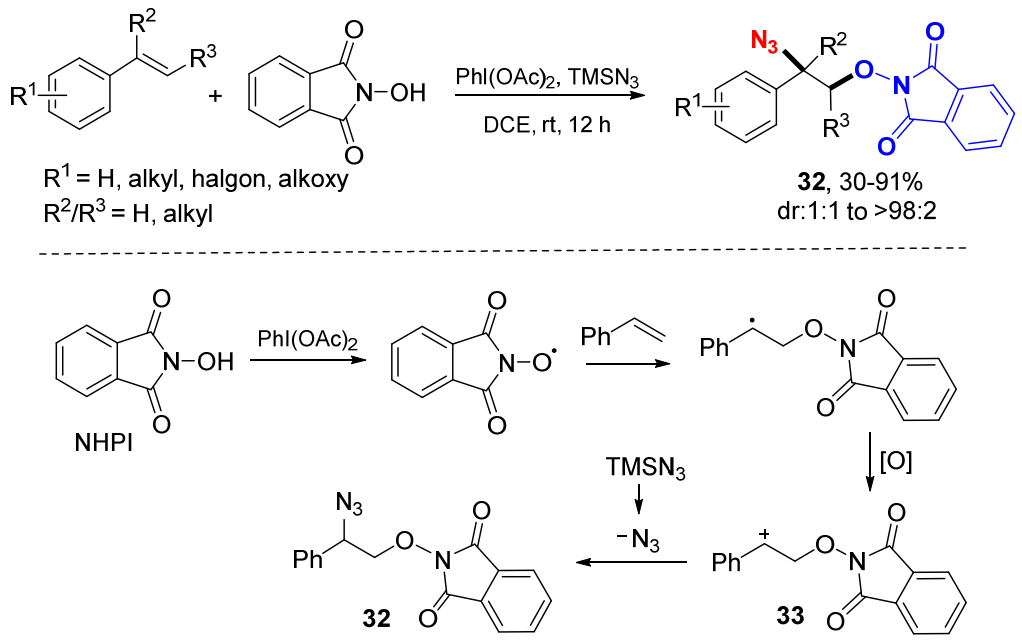

Scheme 18. PINO radical-initiated difunctionalization of alkenes.

The Dagousset group reported an alkoxy radical-initiated difunctionalization reaction of alkenes for the synthesis of functionalized diethers $34 \mathbf{a}$ or amino ethers $34 \mathbf{b}$ under batch or flow reaction conditions (Scheme 19) [40]. A radical/cationic addition mechanism was confirmed by electron paramagnetic resonance (EPR) studies. Alkoxy radicals generated from 4-cyano-substituted $N$-alkoxypyridinium salt under the catalysis of $f a c-\operatorname{Ir}(\mathrm{ppy})_{3}$ add to alkenes followed by the SET of $\operatorname{Ir}(\mathrm{ppy})_{3}{ }^{+}$to form corresponding carbocations. Nucleophilic addition of alcohols or acetonitrile gives difunctionalized products 34 .

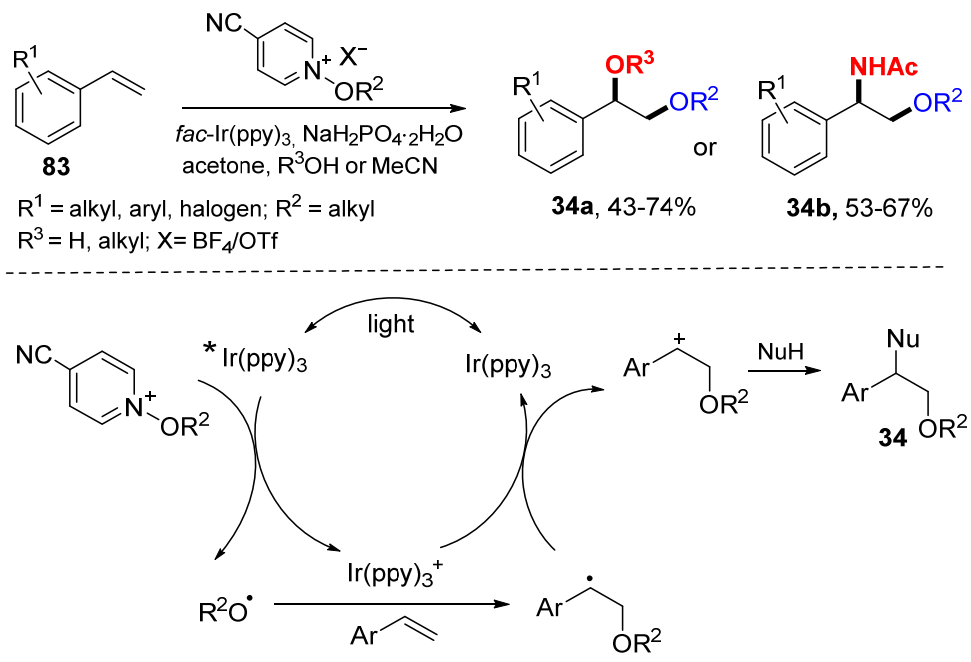

Scheme 19. Dialkoxylation and aminoalkoxylation of alkenes. * excited catalyst.

\section{Phosphorous Radical-Initiated Reactions}

Phosphorous radical-initiated difunctionalization reactions are highlighted in this section. So far we only found reactions of phosphinoyl $\left[\mathrm{R}_{2}(\mathrm{O}) \mathrm{P}^{\prime}\right]$ and phosphonyl $\left[(\mathrm{RO})_{2}(\mathrm{O}) \mathrm{P}^{\prime}\right]$ radicals generated from corresponding phosphine oxides and phosphonates [41].

The Zou and Zhang groups reported Cu-catalyzed difunctionalization of alkenes with diphenylphosphine oxide $\left(\mathrm{HPOPh}_{2}\right)$ and trimethylsilylcyanide (TMSCN) (Scheme 20) [42]. The phosphinoyl radicals generated from the oxidation of $\mathrm{HPOPh}_{2}$ with $\mathrm{Mn}(\mathrm{OAc})_{3}$ react with alkenes which are oxidized with $\mathrm{Cu}^{\mathrm{II}}$ and then cyanylated with $\mathrm{CN}^{-}$to give products 35 . 

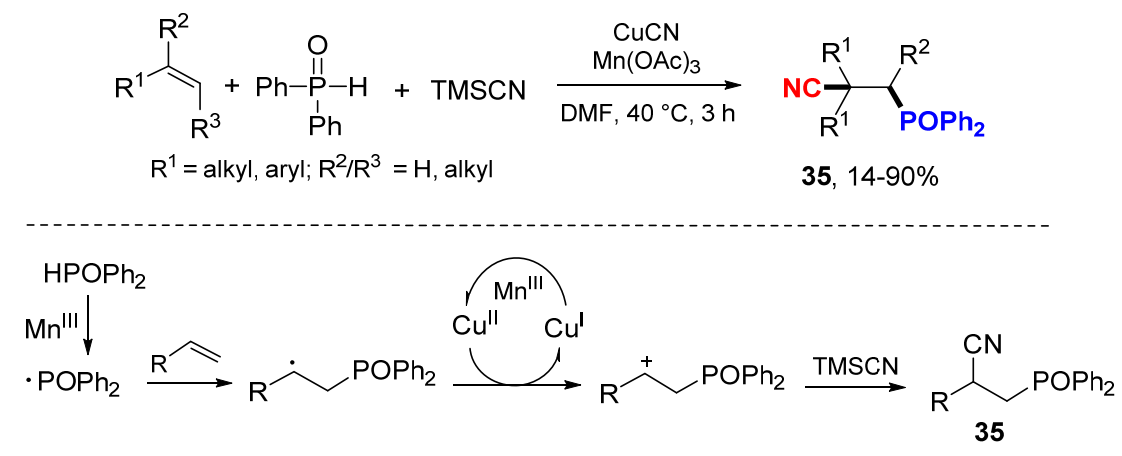

Scheme 20. Phosphinoylation and cyanylation of alkenes.

Wang and coworkers reported Ce-catalyzed phosphinoylation and nitration reactions of alkenes (Scheme 21) [43]. $\mathrm{CAN}\left(\mathrm{NH}_{4}\right)_{2} \mathrm{Ce}\left(\mathrm{NO}_{3}\right)_{6}$ was used as P-radical initiator and also nitrate donor to afford $\beta$-nitrooxyphosphonates 36 . The phosphinoyl radicals generated form phosphine oxides or phosphonates through a SET with $\mathrm{Ce}^{\mathrm{IV}}$ add to alkenes to form benzylic cations after the second SET with CAN. Reaction of benzylic cations with nitrate give products 36 .

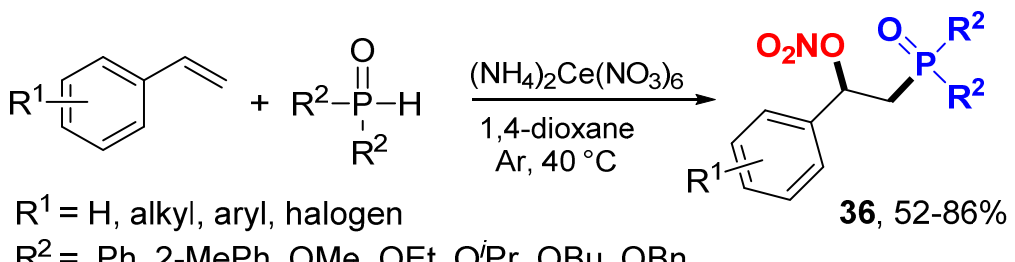

$\mathrm{R}^{2}=\mathrm{Ph}, 2-\mathrm{MePh}, \mathrm{OMe}, \mathrm{OEt}, \mathrm{O}^{\prime} \mathrm{Pr}, \mathrm{OBu}, \mathrm{OBn}$

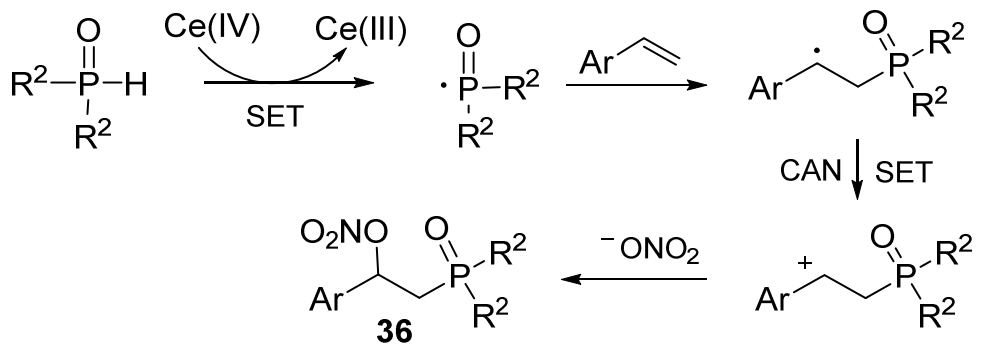

Scheme 21. P-radical initiated difunctionalizations of styrenes.

Fang and coworkers reported $\mathrm{TBHP}$ and $\mathrm{FeCl}_{3}$-promoted difunctionalization of alkenes with phosphine oxides or phosphonates and anilines for the synthesis of products 37 (Scheme 22) [44]. In the reaction with diphenylphosphine oxide, phosphinoyl radicals add to alkenes to form carbocations after oxidation with $\mathrm{FeCl}_{3}$. Trapping of carbocations by amines afford corresponding $\alpha, \beta$-phosphinoamination products 37 .

Using a similar strategy of heteroaryl group migration described in Scheme 14, the Zhu group accomplished phosphinoyl or phosphonyl radical-initiated difunctionalization of $\gamma$-hydroxyalkenes (Scheme 23) [45]. In the presence of TBHP, the P-radicals generated from phosphine oxides or phosphonates add to alkenes followed by 1,4-heteroaryl migration of 39 to form radicals 40 , oxidation of the radicals to cations to give products $\mathbf{3 8}$ after proton transfer. In addition to heteroaryl, other groups such as cyano and imino, could also be used as $\mathrm{R}^{2}$ for the migration. 


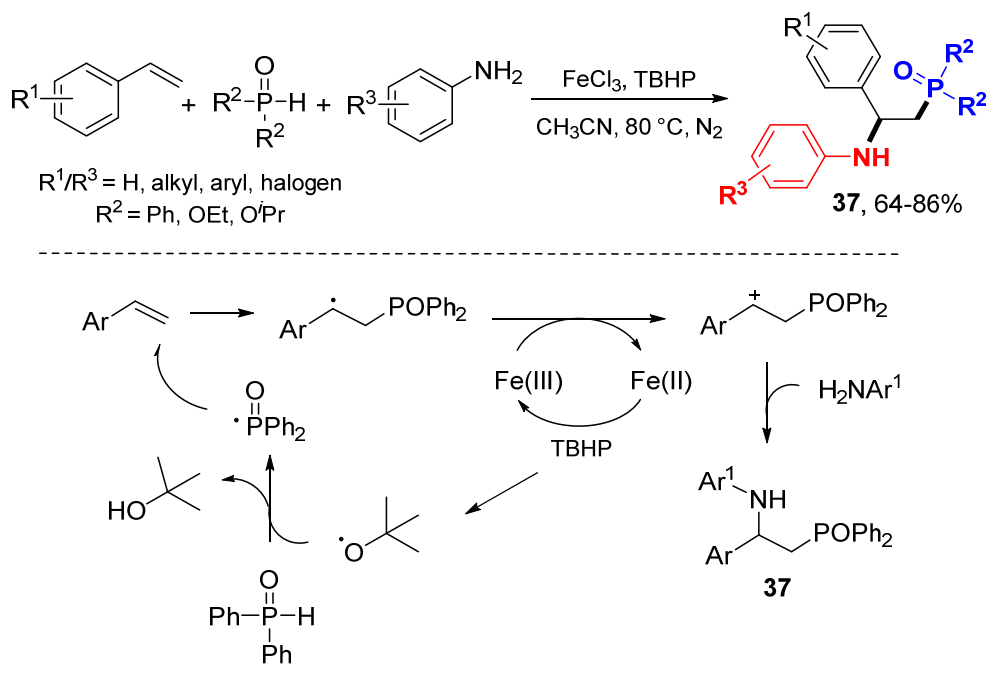

Scheme 22. Phosphinoamination of styrenes.

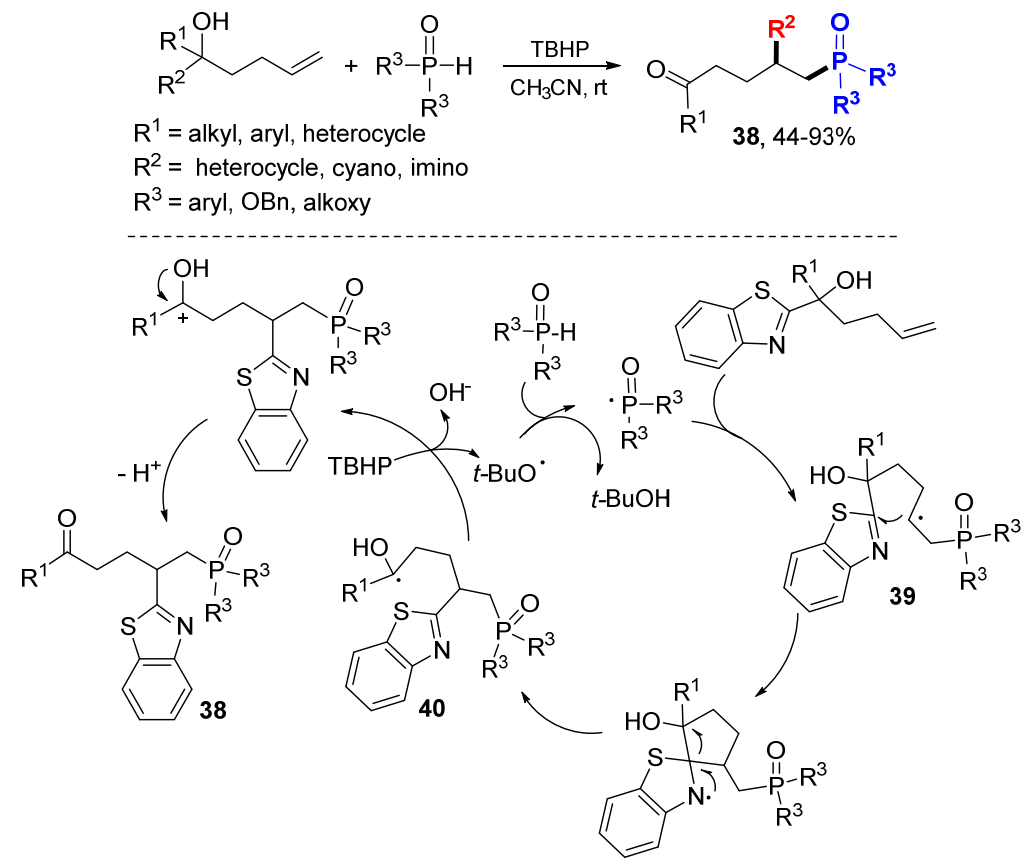

Scheme 23. Radical-mediated phosphinoyl-functionalization of alkenes.

\section{Sulfur Radical-Initiated Reactions}

Sulfur-centered radicals, such as sulfenyl (RS ), sulfonyl $\left[\mathrm{RS}(\mathrm{O}){ }_{2} \mathrm{~S}^{*}\right]$, and thiocyanate $(\mathrm{SCN})$ radicals, have been used for difunctionalization reactions [41]. The sulfur radicals could be generated from corresponding reagents through homolytic N-S bond cleavage, single electron oxidation of KSCN, and electrochemical and photoredox catalytic reactions.

The Wu group reported a method for the synthesis of $\beta$-halo vinylsulfones through $\mathrm{Cu}$ catalyzed reaction of alkynes with aryldiazonium tetrafluoroborates and DABCO $\left(\mathrm{SO}_{2}\right)_{2}$ (Scheme 24A) [46]. Arylsulfonyl radicals 42 generated from the reaction of aryldiazonium cations with DABCO $\left(\mathrm{SO}_{2}\right)_{2}$ attack alkynes to form stable alkenyl radicals which are oxidized to alkenyl cations with $\mathrm{CuCl}$ followed by nucleophilic addition with halides to give $\beta$-halo vinylsulfones 41 . The research group also extended the scope of the reaction of aryl alkenes under photoredox conditions and using alkyl nitriles as nucleophiles to afford aminosulfonylation products 43 (Scheme 24B) [47]. 


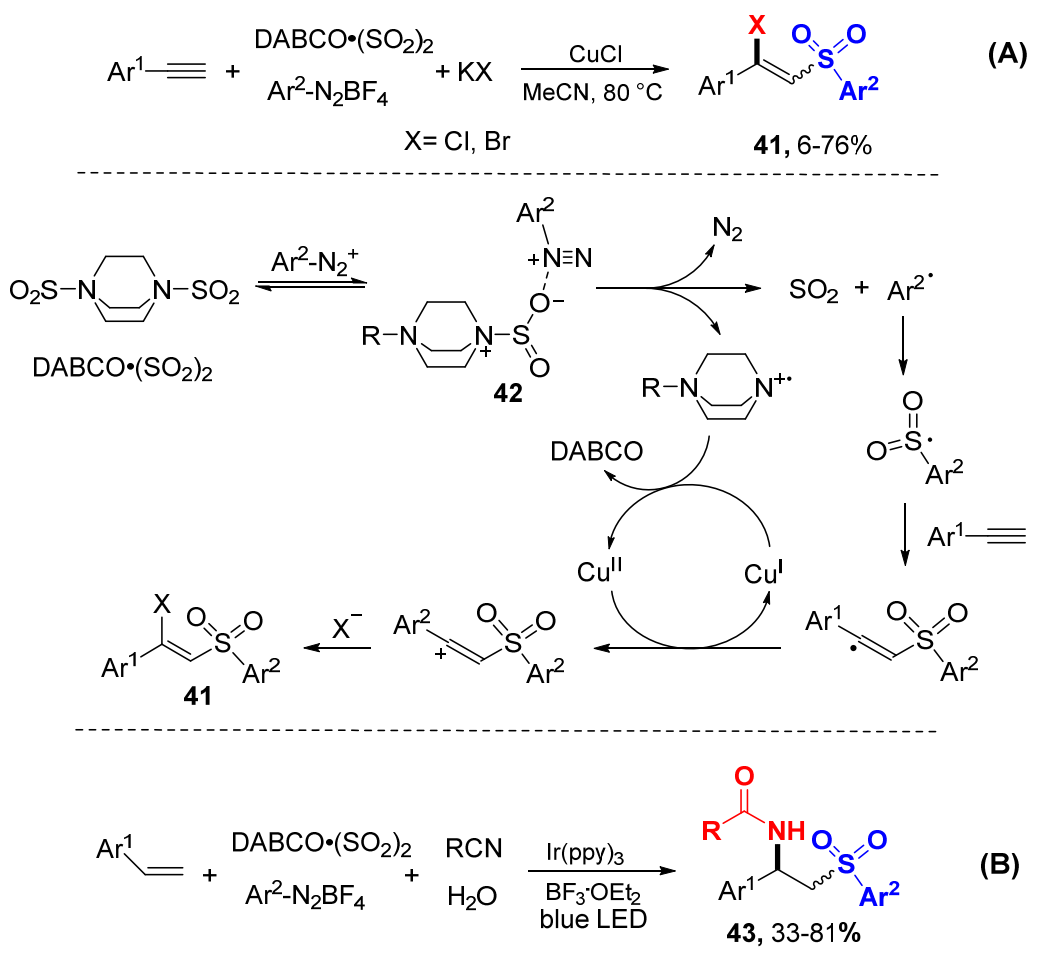

Scheme 24. Halosulfonylation of aryl alkynes. (A) reaction of alkenes. (B) reaction of alkynes.

Majee and coworkers reported a method for dithiocyanation of alkynes and alkenes with $\mathrm{KSCN}$ and $\mathrm{Na}_{2} \mathrm{~S}_{2} \mathrm{O}_{8}$ to give products 44 and 45, respectively (Scheme 25) [48]. In the reaction of alkynes, the thiocyanate radical generated from the oxidation of KSCN adds to alkynes, followed by oxidization and reaction with KSCN to give dithiocyanation alkenes 44. Other than nucleophilic addition of thiocyanate anion, coupling of thiocyanate radical is also a possible pathway.

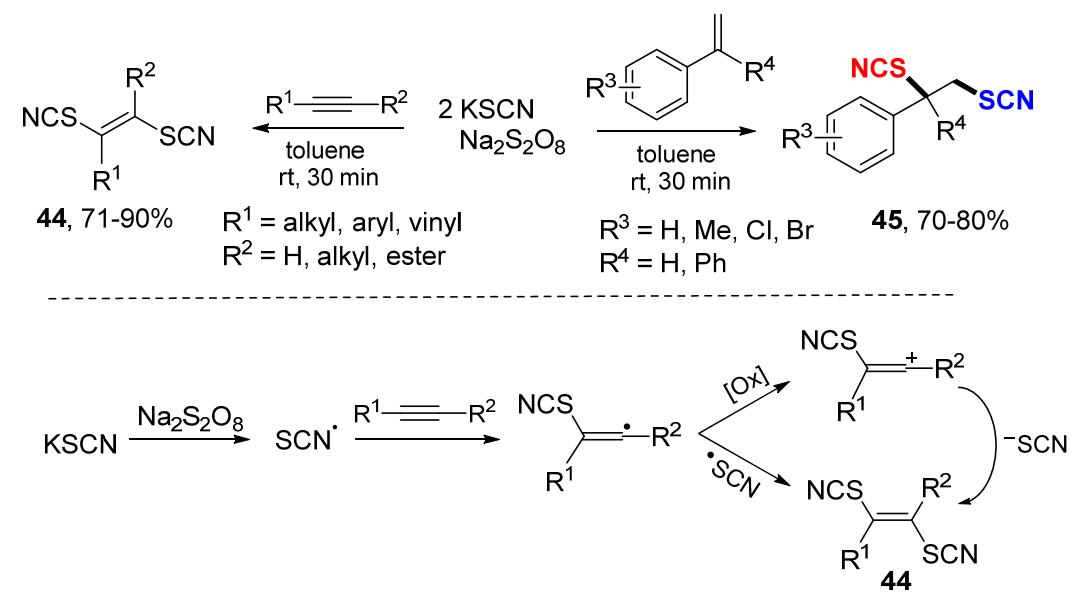

Scheme 25. Dithiocyanation of alkynes and alkenes.

The Han group developed an electrochemical oxysulfuration reaction of styrenes by addition of sufenylradicals and alkoxy nucleophiles (Scheme 26) [49]. The arylsufenyl radicals generated from thiophenol through the SET oxidation at the anode add to alkenes and then oxidize to carbocations at the anode. Trapping of the carbocations by alcohols affords the oxysulfuration products 46 . 


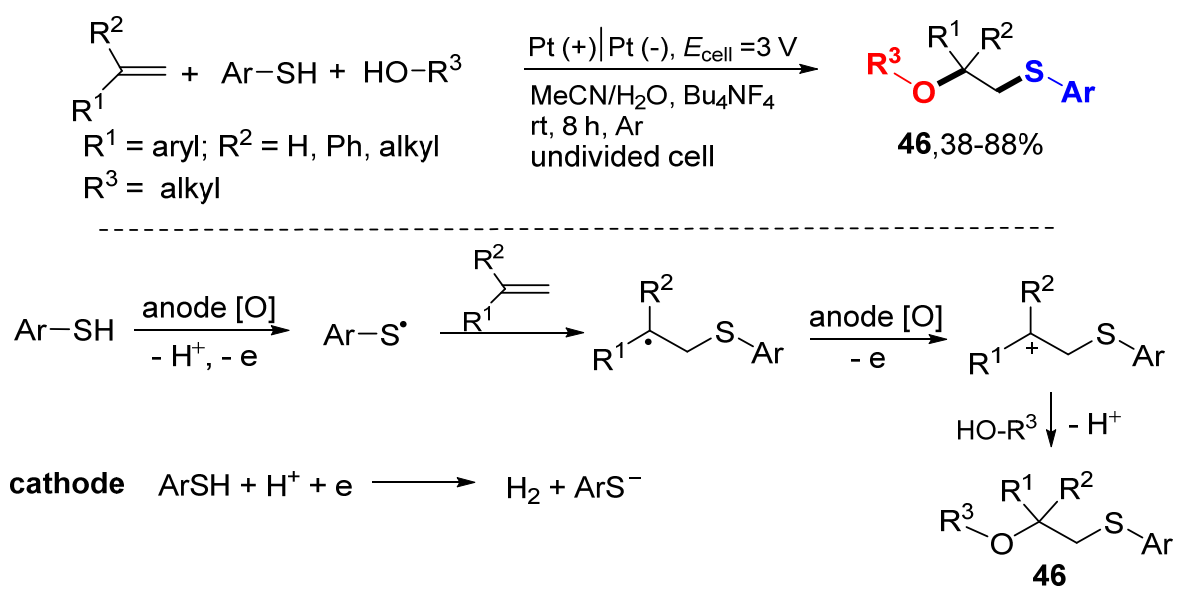

Scheme 26. Electrochemical difunctionalization of styrenes.

Guo and coworker reported electrochemical sulfonylheteroarylation reaction of alkenes involving heteroaryl group migration (Scheme 27) [50]. The arylsulfonyl radicals generated from sulfinic acid at the anode add to benzothiazole ring for heteroaryl migration followed by single-electron oxidation at the anode to afford cations and then products 47 after deprotonation. This ipso-aryl group migration process is similar to those presented in Schemes 14 and 23.

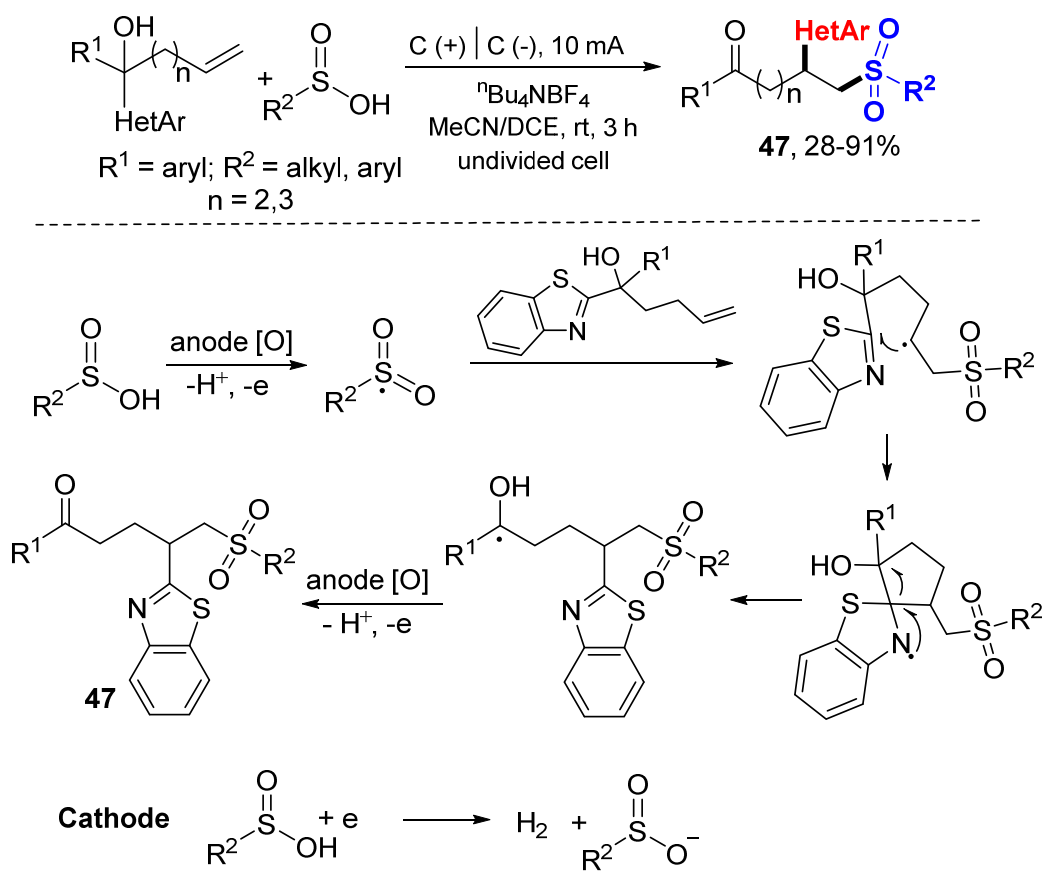

Scheme 27. Electrochemical sulfonylheteroarylation of alkenes.

An electrochemical oxidative alkoxysulfonylation of alkenes with sulfonyl hydrazines and alcohols have been reported by the Lei group (Scheme 28) [51]. The reactions were carried out at room temperature and only molecular nitrogen and hydrogen are released as byproducts. Sulfonyl radicals generated from sulfonyl hydrazines through oxidation at anode react with alkenes and then undergo second oxidation at anode to form benzyl cation intermediates. Nucleophilic attack of cations with alkoxyl anions give alkoxysulfonylation products 48. The alkoxyl anions are generated from alcohols on the cathode. 


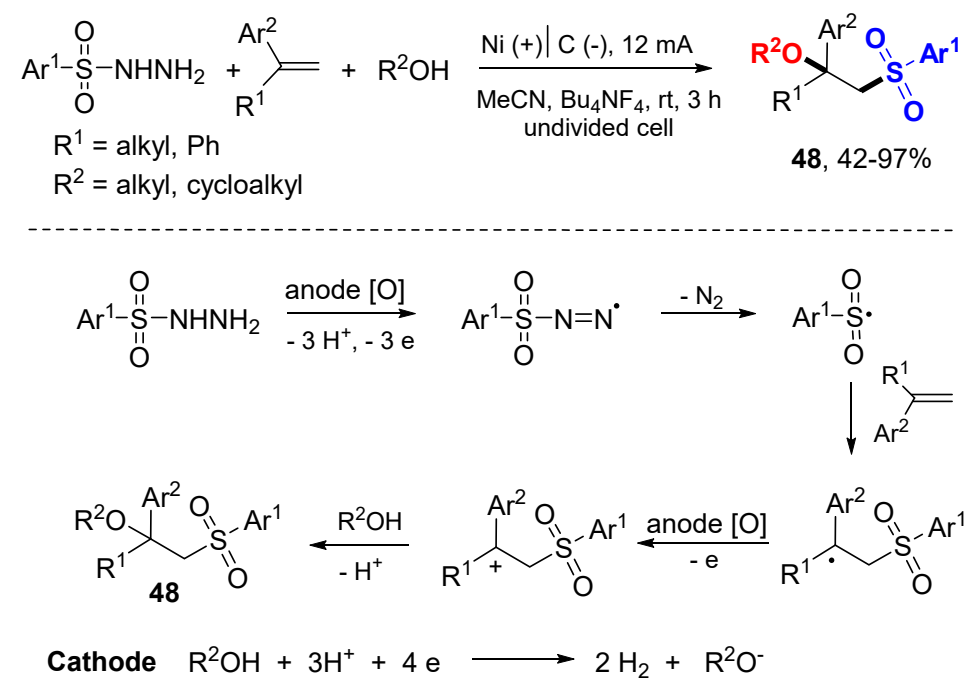

Scheme 28. Electrochemical oxidative alkoxysulfonylation of alkenes.

The Lei group also reported sulfenyl radical-initiated oxysulfenylation and aminosulfenylation of alkenes using thiophenols/thiols as thiolating agents and alcohols/amines as nucleophiles (Scheme 29) [52]. Under electrochemical reaction conditions, thiophenols/thiols are converted to sulfenyl radicals for addition to alkenes followed by anode oxidation of resulting radicals to cations and nucleophilic addition with alcohols or amines to give oxysulfenylation products 49 and aminosulfenylation products 50, respectively.

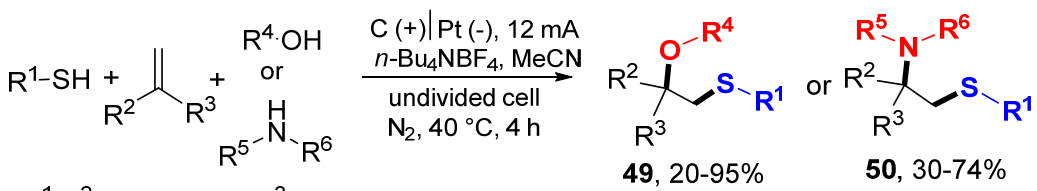

$\mathrm{R}^{1 / 1} \mathrm{R}^{2}=$ alkyl, aryl; $\mathrm{R}^{3}=$ alkyl, heterocycle

49, $20-95 \% \quad 50,30-74 \%$

$\mathrm{R}^{4}=\mathrm{H}, \mathrm{Ac}$, alkyl, alkoxy; $\mathrm{R}^{5}=\mathrm{H}$, alkyl

$\mathrm{R}^{6}=\mathrm{Ac}$, alkyl, aryl

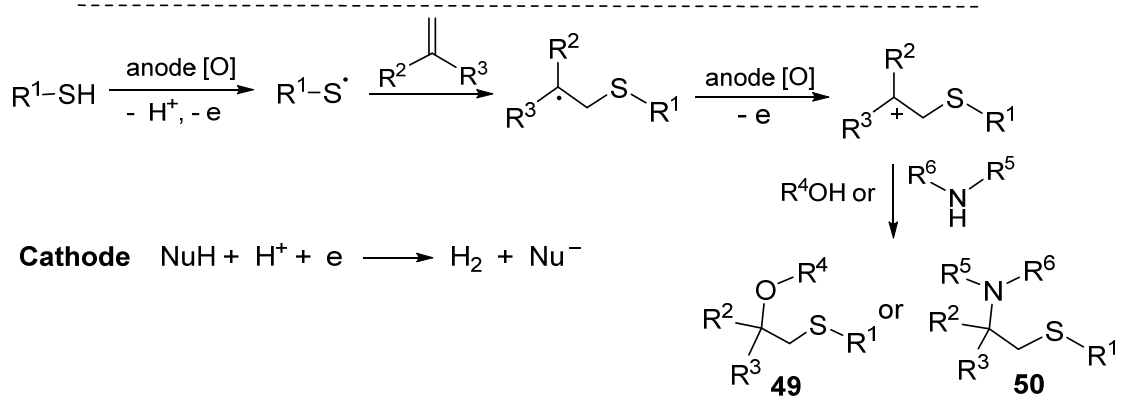

Scheme 29. Electrochemical oxysulfenylation and aminosulfenylation of alkenes.

Han and coworkers reported a photoredox-catalyzed chlorosulfonylation reaction of alkynes using sulfonyl chlorides as both sulfonyl radical and chlorine sources (Scheme 30) [53]. A variety of $(E)$-selective $\beta$-chlorovinyl sulfones $\mathbf{5 1}$ were prepared from terminal and internal alkynes.

The Nie and Niu group reported the preparation of $\beta$-ketosulfones 52 via graphitic carbon nitride ( $\mathrm{p}-\mathrm{g}-\mathrm{C}_{3} \mathrm{~N}_{4}$ )-photocatalyzed hydrosulfonylation of alkynes in aerobic aqueous medium (Scheme 31) [54]. The heterogeneous semiconductor is recyclable at least 6 times without significant reducing activity. Arylsulfonyl radicals generated from the complex of DABCO $\left(\mathrm{SO}_{2}\right)_{2}$ and $\mathrm{Ar}^{1}-\mathrm{N}_{2} \mathrm{BF}_{4}$ add to arylalkynes to form arylalkenyl radicals and then arylalkenyl cations after SET oxidation. Hydrolysis of the cations give $\beta$-keto sulfones 52 . 


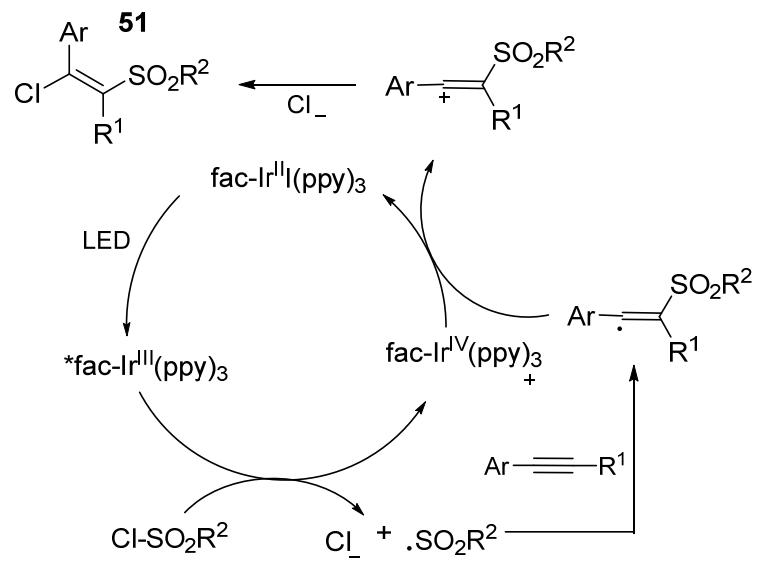

$\mathrm{R}^{1}=\mathrm{H}$, alkyl; $\mathrm{R}^{2}=$ aryl, alkyl

51, $42-90 \%$

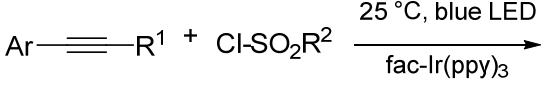<smiles>[R1]/C(=C(\Cl)Br)S([R20])=O</smiles>

Scheme 30. Chlorosulfonylation of arylalkynes. * excited catalyst.

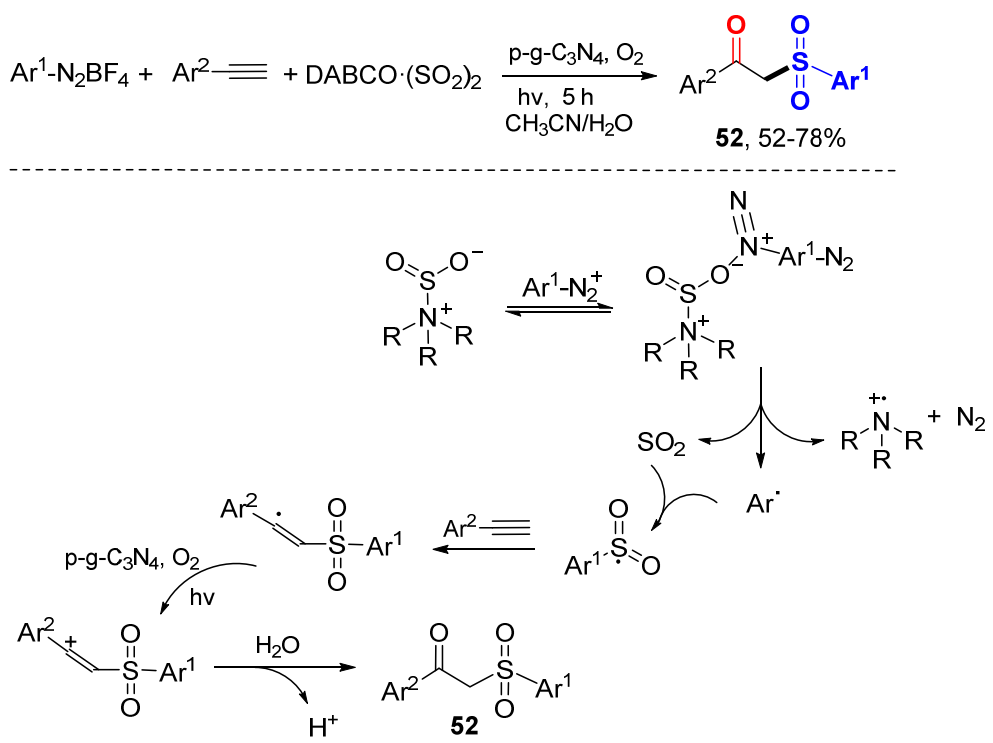

Scheme 31. Light-promoted hydrosulfonylation of arylalkynes.

The Zhu group reported photoredox heteroarylsulfonylation and oximinosulfonylation of unactivated alkenes via sulfonyl radical addition followed by heteroaryl or oximino group migration and oxidation of hydroxyl to ketone to afford products 53 (Scheme 32) [55]. The reaction process is similar to that presented in Scheme 14.

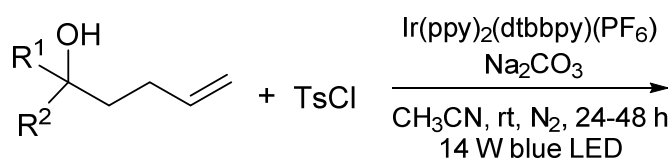

$\mathrm{R}^{1}=$ alkyl, aryl; $\mathrm{R}^{2}=$ hetAr, oximine

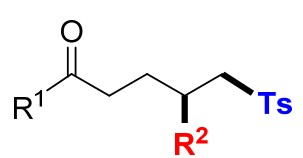

53, 38-96\%

Scheme 32. Heteroarylsulfonylation and oximinosulfonylation of alkenes. 


\section{Fluorocarbon Radical-Initiated Reactions}

Fluorine atom and fluorinated alkyl groups could be used to improve molecules' stability, bioavailability, and other physical, chemical and biological properties [56]. The development of new synthetic methods to introducing flouring-containing groups, such as $\mathrm{CF}_{2} \mathrm{R}$, $\mathrm{CF}_{3}$ and multi-carbon perflourinated alkyls $\left(\mathrm{R}_{\mathrm{f}}\right)$, is a topic of current interest in both organic and medicinal chemistry [57]. Radical-initiated difunctionalization is a good approach for the synthesis of fluorinated compounds [58]. Most fluorocarbon radicals presented in this section are generated by metal-catalyzed photo-redox or electrochemical reactions from the substrates including Umemoto's [59], Togni's [60], and Langlois' $\left(\mathrm{CF}_{3} \mathrm{SO}_{2} \mathrm{Na}\right)$ reagents. Perfluoroalkanesulfonyl chloride $\left(\mathrm{CF}_{3} \mathrm{SO}_{2} \mathrm{Cl}\right), \alpha$-bromo-2,2-difluoroacetates, bromodifluoromethylphosphonates, and perfluoroalkyl-containing hypervalent iodines are also good sources for fluorocarbon radicals.

The Koike and Akita group developed a photoredox catalyzed oxytrifluoromethylation reaction of alkenes using Umemoto's reagent as the $\mathrm{CF}_{3}$ source (Scheme 33) [61]. In this catalytic system, the sunlight is an efficient light source. The reaction process includes the generation of a $\mathrm{CF}_{3}$ radical from Umemoto's reagent by photo catalytic $\mathrm{SET}$, addition of $\mathrm{CF}_{3}$ radical to alkenes to form intermediate carbon radicals, oxidation to carbocation intermediates by catalytic SET, and alcohol nucleophilic addition give products 54 .

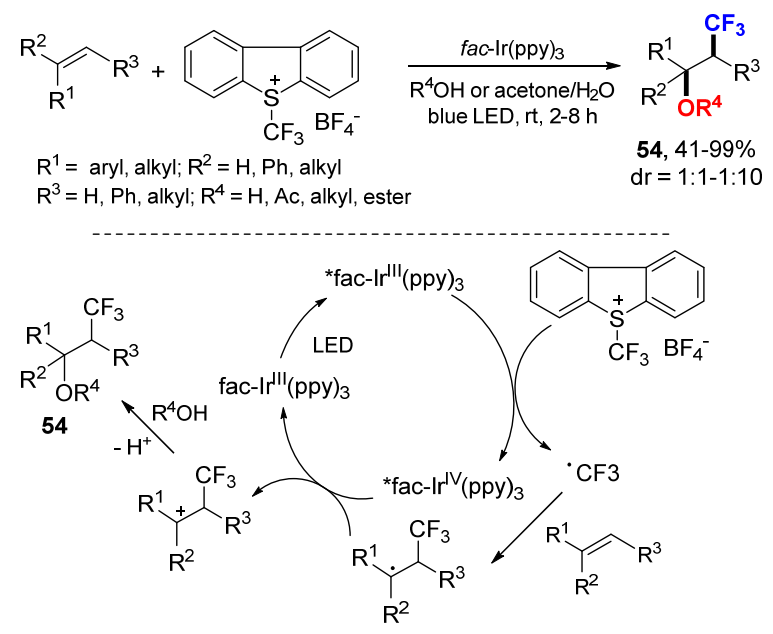

Scheme 33. Photoredox catalyzed oxytrifluoromethylation of alkenes. * excited catalyst.

The Koike and Akita group also reported photoredox reaction of alkenes using Umemoto's reagent as $\mathrm{CF}_{3}$ source and $\mathrm{Ru}(\mathrm{bpy})_{3}\left(\mathrm{PF}_{6}\right)_{2}$ as a catalyst for the synthesis of aminotrifluoromethylation products 55 (Scheme 34A) [62]. The Magnier and Masson group used the similar reaction conditions in the synthesis of $\beta$-trifluoromethylated azides $56 \mathbf{a}$ and amines 56b (Scheme 34B) [63].

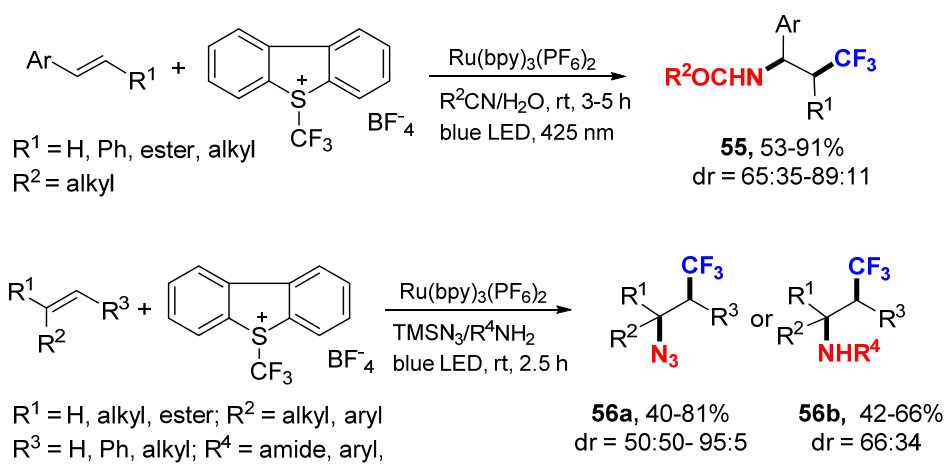

Scheme 34. Difunctionalization using Umemoto's reagent. (A) reaction with $\mathrm{R}_{2} \mathrm{CN} / \mathrm{H}_{2} \mathrm{O}$. (B) reaction with $\mathrm{TMSN}_{3} / \mathrm{R}_{4} \mathrm{NH}_{2}$. 
The She and Li group reported acyloxytrifluoromethylation of alkenes with the Umemoto II reagent using $\mathrm{Ru}(\mathrm{bpy})_{3}\left(\mathrm{PF}_{6}\right)_{2}$ as a photoredox catalyst (Scheme 35) [64]. N,NDimethylformamide (DMF) was employed as a solvent and also an acetylation reagent. $\mathrm{CF}_{3}$ radical generated from the Umemoto's reagent through catalytic SET adds to arylalkenes to give stable benzyl radicals which are oxidized to benzyl carbocations followed by nucleophilic addition of DMF to give imine intermediates and then hydrolyzed to give desired products 57 .

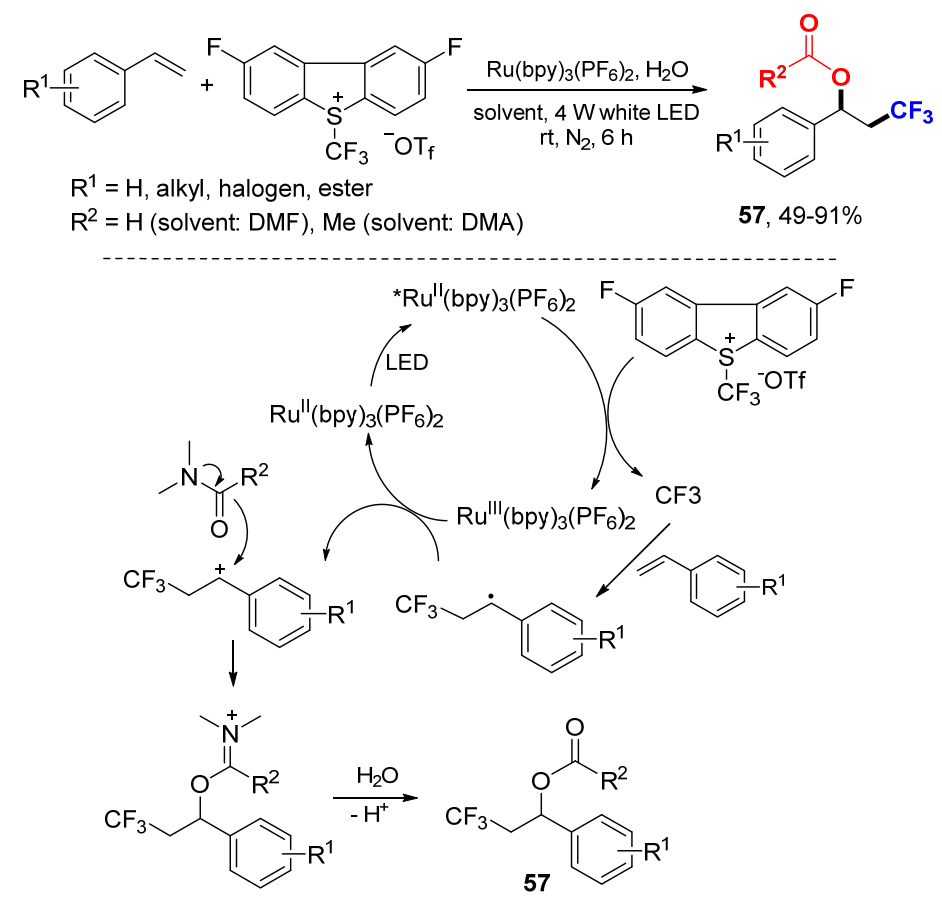

Scheme 35. Acyloxytrifluoromethylation of styrenes. ${ }^{*}$ excited catalyst.

The Koike and Akita group reported trifluoromethylation-initiated reaction for the synthesis of $\alpha$-trifluoromethylated ketones 58 (Scheme 36) [65]. $\mathrm{CF}_{3}$ radical generated from the Togni's reagent II through photocatalytic reductive SET of $f a c-\left[\operatorname{Ir}^{\mathrm{III}}(\mathrm{ppy})_{3}\right]$ adds to alkenes followed by oxidative SET gave $\beta-\mathrm{CF}_{3}$-substituted carbocationic intermediates. Nucleophilic attack of DMSO to carbocations affords alkoxysulfonium intermediates which then react with $o$-iodobenzoate for Korblum oxidation to give final products 58 .

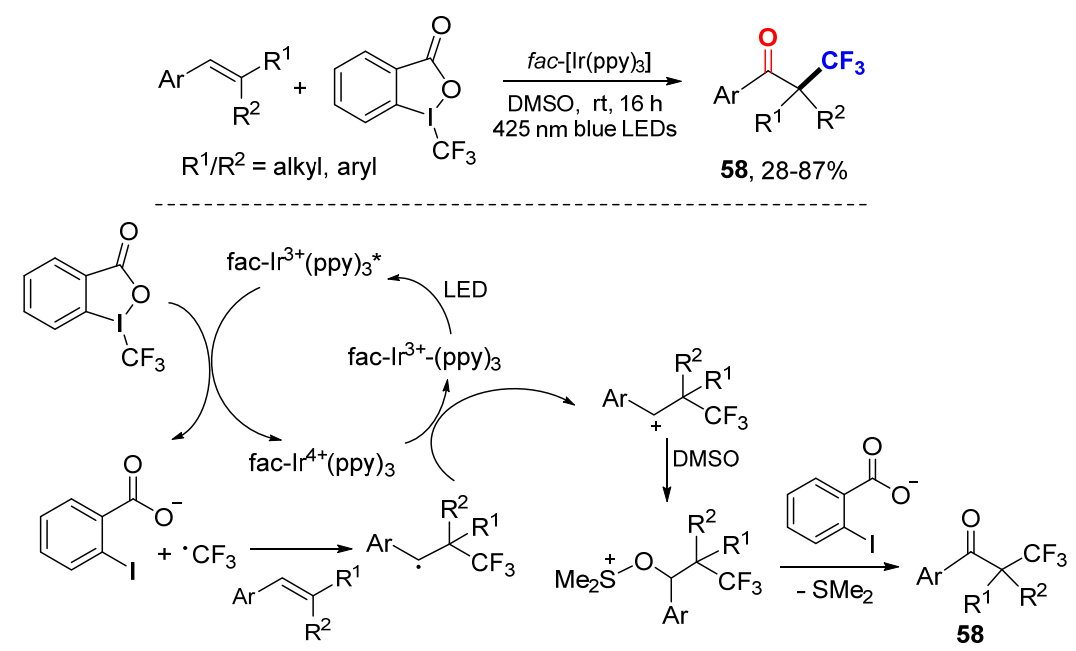

Scheme 36. Photocatalytic keto-trifluoromethylation of styrenes. * excited catalyst. 
The Magnier and Masson group reported a photoredox-catalyzed carbotrifluoromethylation of enecarbamates using Togni's reagent II as the $\mathrm{CF}_{3}$ source and various $\mathrm{O}_{-}, \mathrm{N}-$, and C-containing compounds as nucleophiles (Scheme 37) [66]. The $\mathrm{CF}_{3}$ radical generated from Togni's reagent by reductive photoredox SET adds to enecarbamates to form $\alpha$-amido radicals which are quickly oxidized to acyliminium cations by a SET process. Nucleophilic trapping by alcohol, $\mathrm{NaN}_{3}$, or $\mathrm{KCN}$ affords the corresponding trifluoromethylated adducts $\mathbf{5 9 a - c}$.

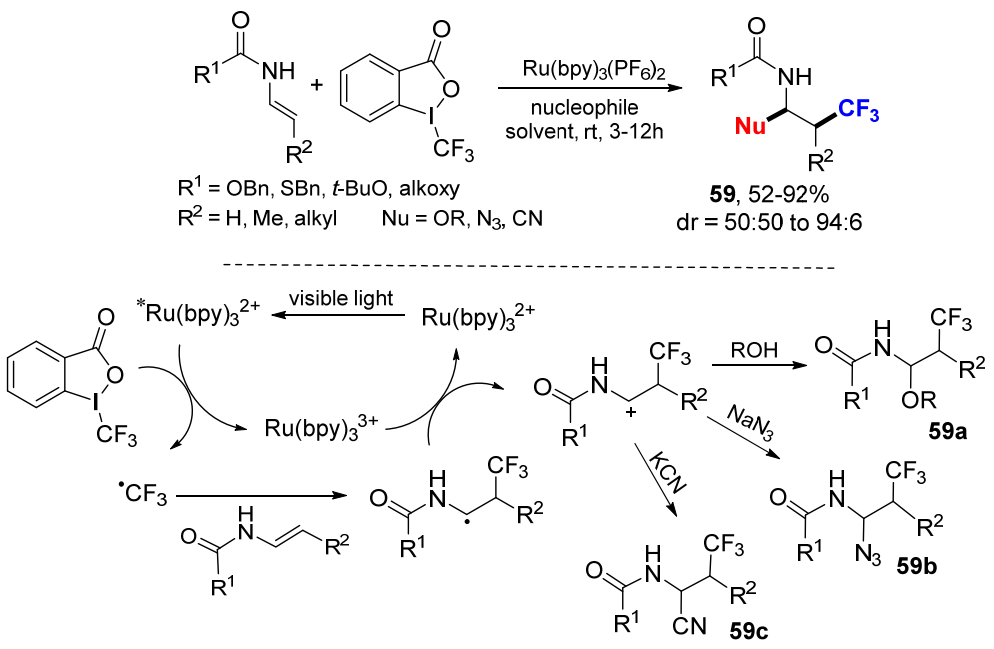

Scheme 37. Difunctionalization of enecarbamates. * excited catalyst.

The Lei group reported an electrochemical reaction for oxytrifluoromethylation and aminotrifluoromethylation of alkenes using sodium trifluoromethanesulfinate $\left(\mathrm{CF}_{3} \mathrm{SO}_{2} \mathrm{Na}\right)$ as the source of $\mathrm{CF}_{3}$ radical (Scheme 38) [67]. $\mathrm{CF}_{3} \mathrm{SO}_{2} \mathrm{Na}$ is oxidized at the anode via SET to afford $\mathrm{CF}_{3}$ radical which adds to styrenes to generate benzylic radicals followed by oxidation to carbocations. Subsequent nucleophilic attack on the carbocations produces desired products 60 .

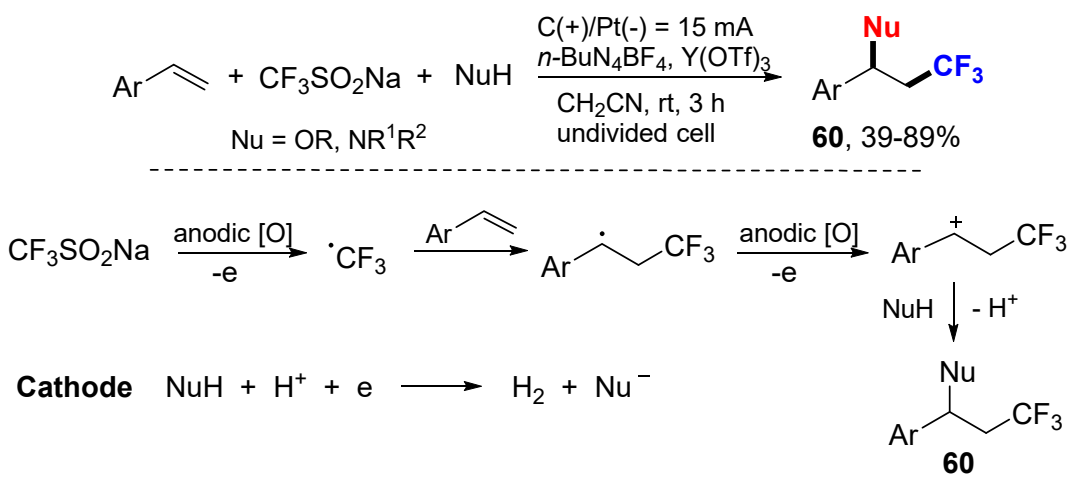

Scheme 38. Trifluoromethylative Difunctionalization of Alkenes Using $\mathrm{CF}_{3} \mathrm{SO}_{2} \mathrm{Na}$.

Han and coworkers reported a photoredox catalytic reaction for 1,2-chlorotrifluoromethylation of alkenes using $\mathrm{CF}_{3} \mathrm{SO}_{2} \mathrm{Cl}$ as a source for $\mathrm{CF}_{3}$ radical and chloride ion (Scheme 39A) [68]. The $\mathrm{CF}_{3}$ radical generated from $\mathrm{CF}_{3} \mathrm{SO}_{2} \mathrm{Cl}$ through reductive $\mathrm{SET}$ of $\mathrm{Ru}$ catalyst add to alkenes, followed by oxidative SET to carbocations and nucleophilic addition of chloride to give chlorotrifluoromethylated products 61. The Han group extended the scope of the reaction for alkynes to make vicinal chlorotrifluoromethylated alkenes $\mathbf{6 2}$ using Ir-based photoredox catalyst (Scheme 39B) [69]. The resultant trifluoromethyl substituted vinyl chlorides can be used for Suzuki coupling to make 1,1-bis-arylalkenes. 


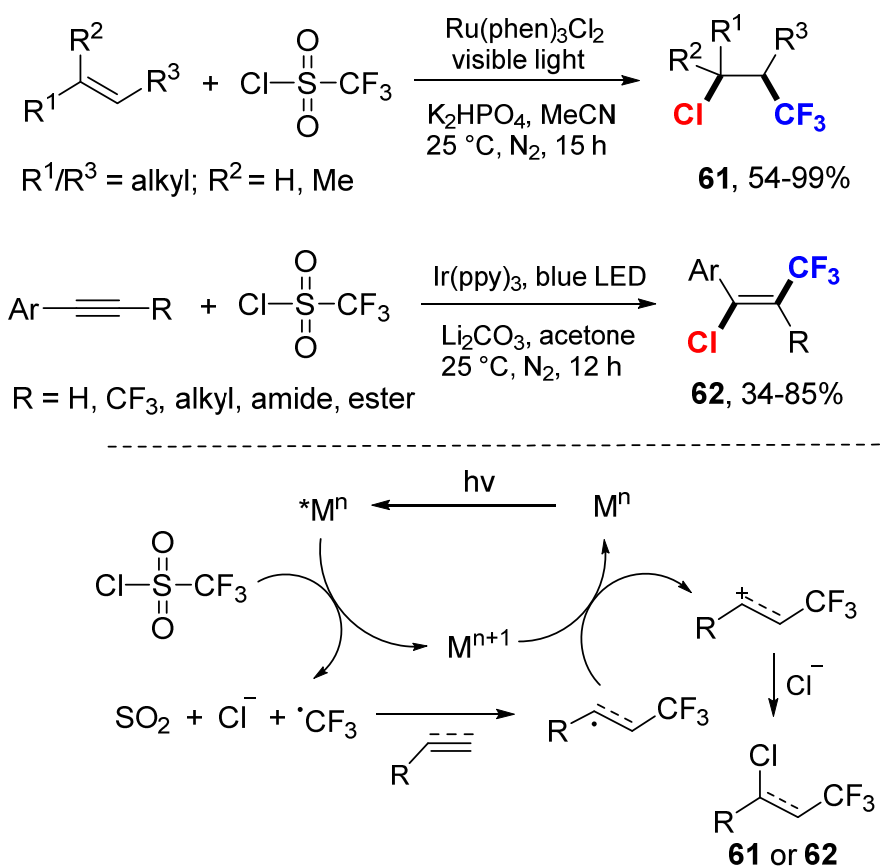

(A)

Scheme 39. Chlorotrifluoromethylation of alkenes and alkynes. (A) $\mathrm{Ru}(\mathrm{phen})_{3} \mathrm{Cl}_{2}$-catalyzed reaction. (B) $\operatorname{Ir}($ ppy)3-catalyzed reaction. * excited catalyst.

The Zhu group applied photoredox catalysis reaction for difluoroalkylarylation of styrenes using $\alpha$-carbonyl difluoroalkyl bromides for $\mathrm{CF}_{2} \mathrm{R}$ radicals and indoles as nucleophiles in the synthesis of difluorolated indole derivatives 63 (Scheme 40) [70]. The $\mathrm{CF}_{2} \mathrm{R}$ radicals generated from $\alpha$-carbonyl difluoroalkyl bromides through reductive SET with Ir-photocatalyst add to alkenes followed by oxidative SET to cations and then react with indoles to give difluoroalkylated products 63 after base-mediated deprotonation.

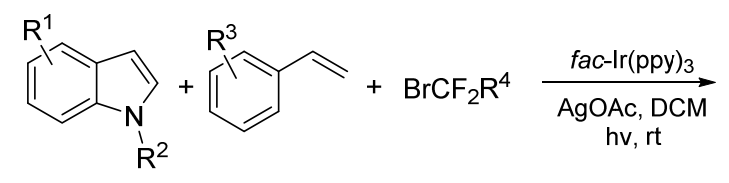

$\mathrm{R}^{1}=\mathrm{H}$, alkyl, alkoxy, halogen, $\mathrm{CN}$, ester

$\mathrm{R}^{2}=\mathrm{H}, \mathrm{Me}, \mathrm{Bn}, \mathrm{Ts}, \mathrm{Boc} ; \mathrm{R}^{3}=4-\mathrm{OMe}, 2-\mathrm{OMe}, 4-\mathrm{Me}$, alkyl; $\mathrm{R}^{4}=$ ester, amide ketone

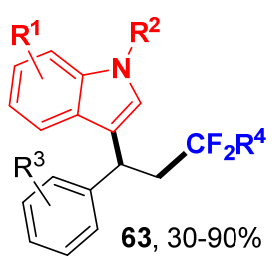

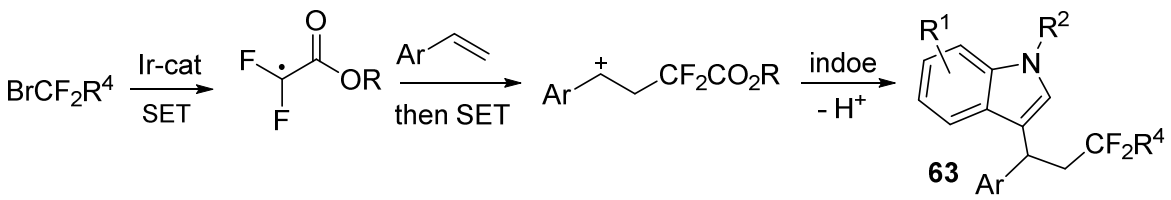

Scheme 40. Photoredox difluoroalkylative difunctionalization of alkenes.

Yang and coworkers reported a photocatalyzed reaction for aminodifluoromethylphosphonation of alkenes using bromodifluoromethylphosphonates as the source of $\mathrm{CF}_{2}$ radical (Scheme 41) [71]. The NaI was used as additive to stabilize the carbocation intermediates and increase the product yields. The difluorocarbon radicals produced through the SET process of bromodifluoromethylphosphonates add to alkenes followed by SET oxidation to cations for nucleophilic addition with amines to give the products 64 after deprotonation. 


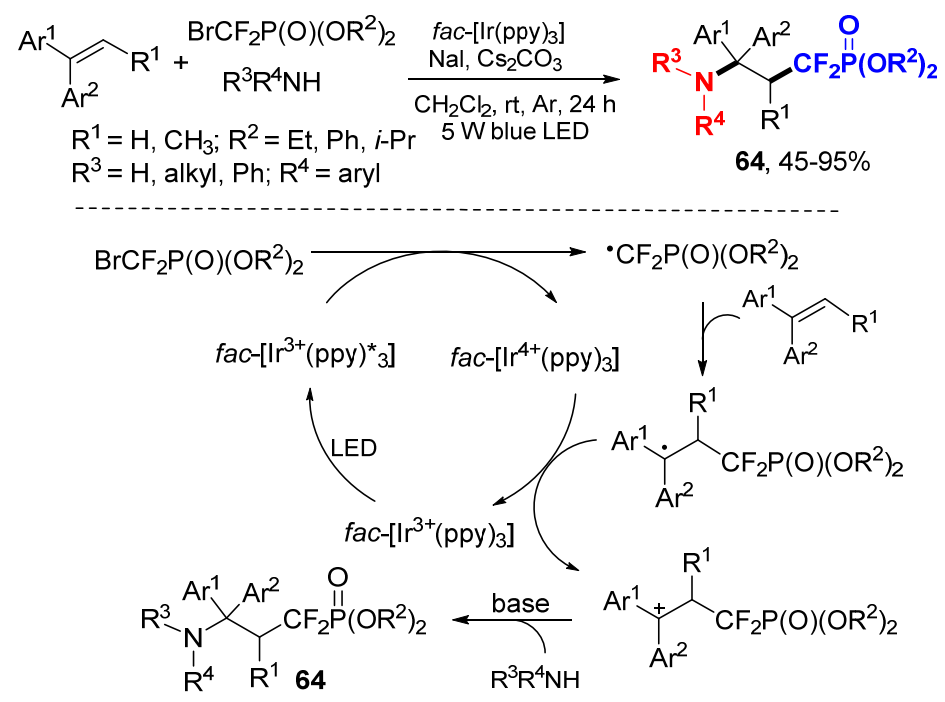

Scheme 41. Photocatalyzed aminodifluoromethylation of alkenes. * excited catalyst.

The Koike and Akita group reported a photocatalytic oxydifluoromethylation of alkenes using shelf-stable and easy-to-handle $N$-tosyl-S-difluoromethyl-S-phenylsulfoximine as a $\mathrm{CF}_{2} \mathrm{H}$ source for the synthesis of $\beta-\mathrm{CF}_{2} \mathrm{H}$-substituted products 65 including alcohols, ethers, and an ester (Scheme 42) [72]. The $\mathrm{CF}_{2} \mathrm{H}$ radical generated from $\mathrm{N}$-tosyl-Sdifluoromethyl-S-phenylsulfoximine by SET process reacts with alkenes to afford carbocationic intermediates after oxidative SET with $\mathrm{Ir}^{(\mathrm{IV})}$ and then solvolysis with $\mathrm{ROH}$ to produce oxydifluoromethylated products 65.

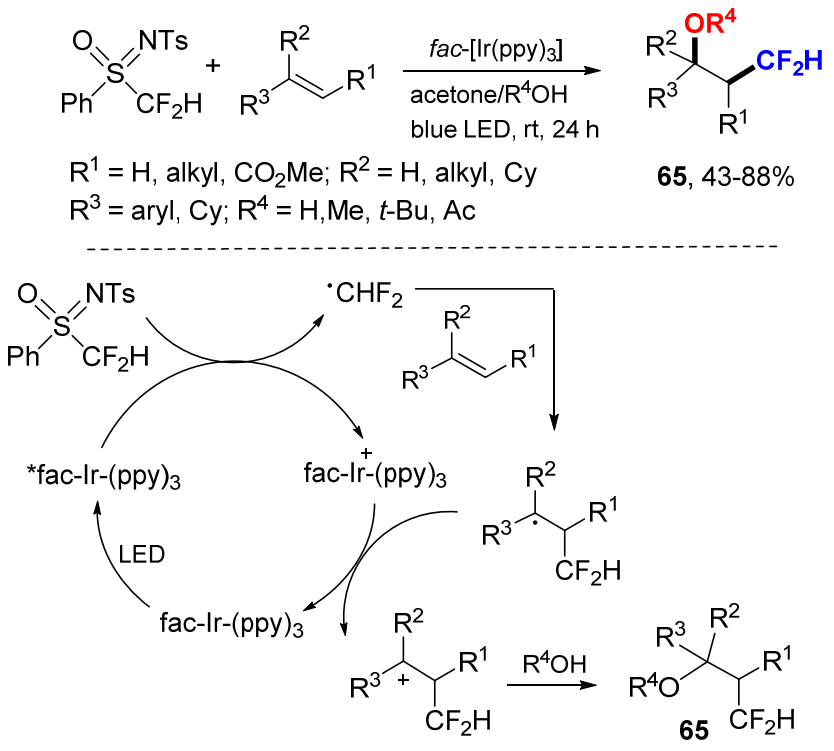

Scheme 42. Photocatalytic oxydifluoromethylation. * excited catalyst.

The Qing group developed a photoredox-catalyzed bromodifluoromethylation reaction of alkenes using difluoromethyltriphenylphosphonium bromide for generating $\mathrm{CF}_{2} \mathrm{H}$ radical and also brominated anion as a nucleophile (Scheme 43A) [73]. The bromonated products 66 can be converted to $\mathrm{CF}_{2} \mathrm{H}$-containing alkenes via an elimination process. The research group also reported a oxydifluoromethylation reaction of styrenes using difluoromethyltriphenylphosphonium bromide as a radical source and alcohols/water as nucleophiles for the synthesis of $\mathrm{CF}_{2} \mathrm{H}$-containing alcohols and ethers 67 (Scheme 43B) [74]. 


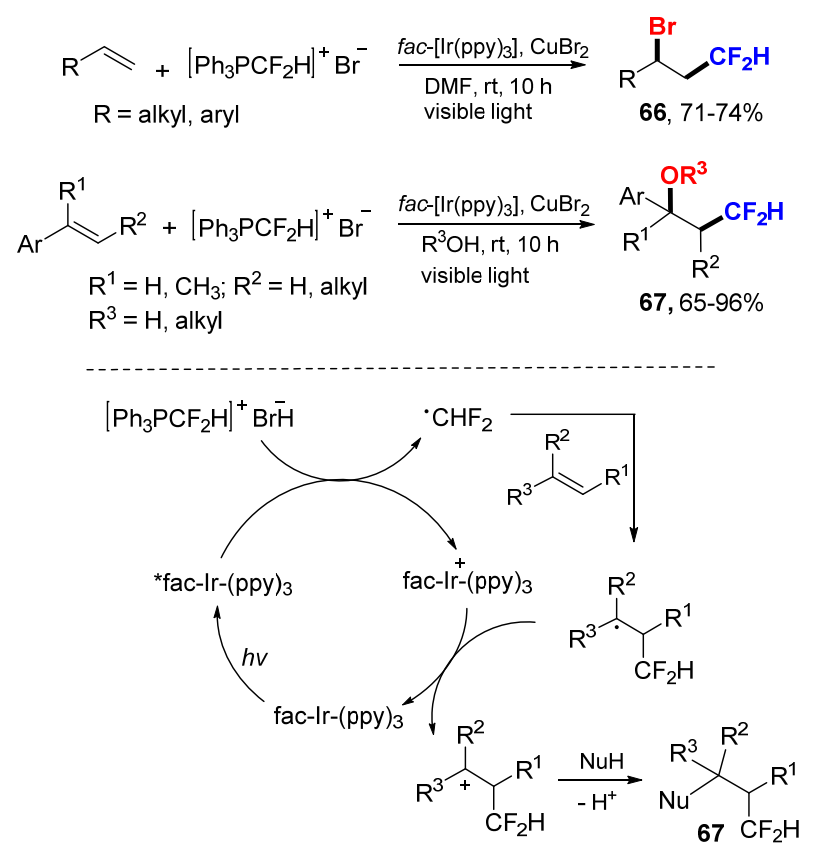

Scheme 43. Bromodifluoromethylation and oxydifluoromethylation of alkenes. (A) reaction with $\mathrm{Br}^{-}$ as nucleophile. (B) reaction with $\mathrm{R}^{3} \mathrm{OH}$ as nucleophile. ${ }^{*}$ excited catalyst.

The Magnier and Masson group employed S-perfluoroalkyl sulfilimino iminium ions 69 as a source of $\mathrm{R}_{\mathrm{f}}$ radicals for oxyperfluoroalkylation of alkenes under photoredox catalysis conditions (Scheme 44) [75]. These stable perfluoroalkyl reagents containing $\mathrm{CF}_{3}$, $\mathrm{C}_{4} \mathrm{~F}_{9}, \mathrm{CF}_{2} \mathrm{Br}$, or $\mathrm{CFCl}_{2}$ can be readily prepared at gram scale from corresponding sulfoxides. The spin-trapping/electron paramagnetic resonance experiments confirmed that key radical intermediates involved in the radical/cationic process. $\mathrm{R}_{\mathrm{f}}$ radicals generated from 69 by reductive SET add to alkenes to form stabilized benzylic radicals and then corresponding carbocations after oxidative SET. Final trapping with methanol provides corresponding products 68 .

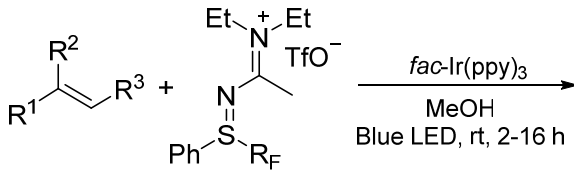

$\mathrm{R}^{1}=$ aryl, alkoxy,carbamate, amide $\mathrm{R}^{2} / \mathrm{R}^{3}=\mathrm{H}, \mathrm{Me} ; \mathrm{RF}_{\mathrm{F}}=\mathrm{CF}_{3}, \mathrm{C}_{4} \mathrm{~F}_{9}, \mathrm{CF}_{2} \mathrm{Br}, \mathrm{CFCl}_{2}$<smiles>[R]C(F)C([R])([R])OC</smiles>

68, $52-88 \%$

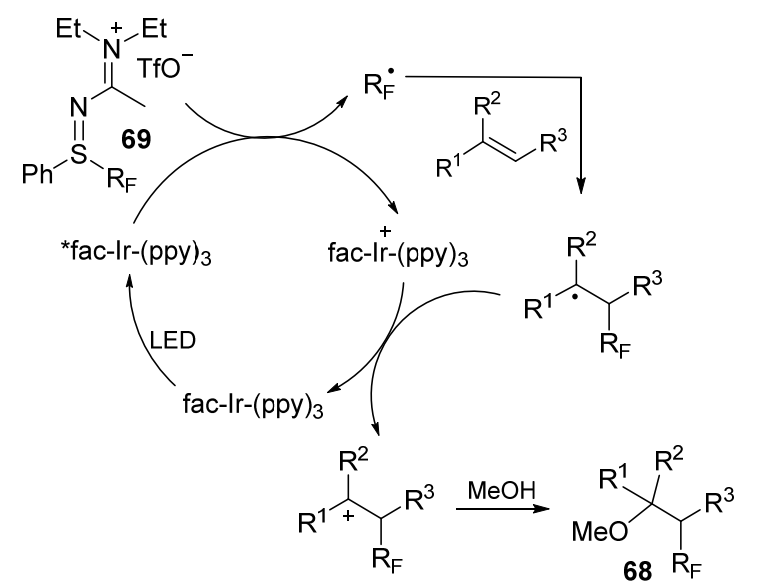

Scheme 44. Oxyperfluoroalkylation with sulfilimino iminium ions. ${ }^{*}$ excited catalyst. 
The Zhu group developed a visible light-promoted difluoroalkylarylation reaction of allylic alcohols for the synthesis of difluoro 1,5-dicarbonyl compounds 70 through radical addition and 1,2-aryl migration process (Scheme 45) [76]. The initial fluorocarbon radicals generated from ethyl 2-bromo-2,2-difluoroacetate or $\mathrm{CF}_{3} \mathrm{I}$ under SET add to allylic alcohols followed by ipso 1,2-aryl migration via spiro[2.5]octadienyl radicals $\mathbf{7 1}$ to give hydroxycarbon radicals. Oxidative SET of the radicals to corresponding carbocations followed by deprotonation give products 70. The Zhu group extended the scope of the reaction for 1,4-aryl and other group migrations using $\beta$-hydroxy alkenes as starting materials for the synthesis of $72 a$ and $72 b$ [77].

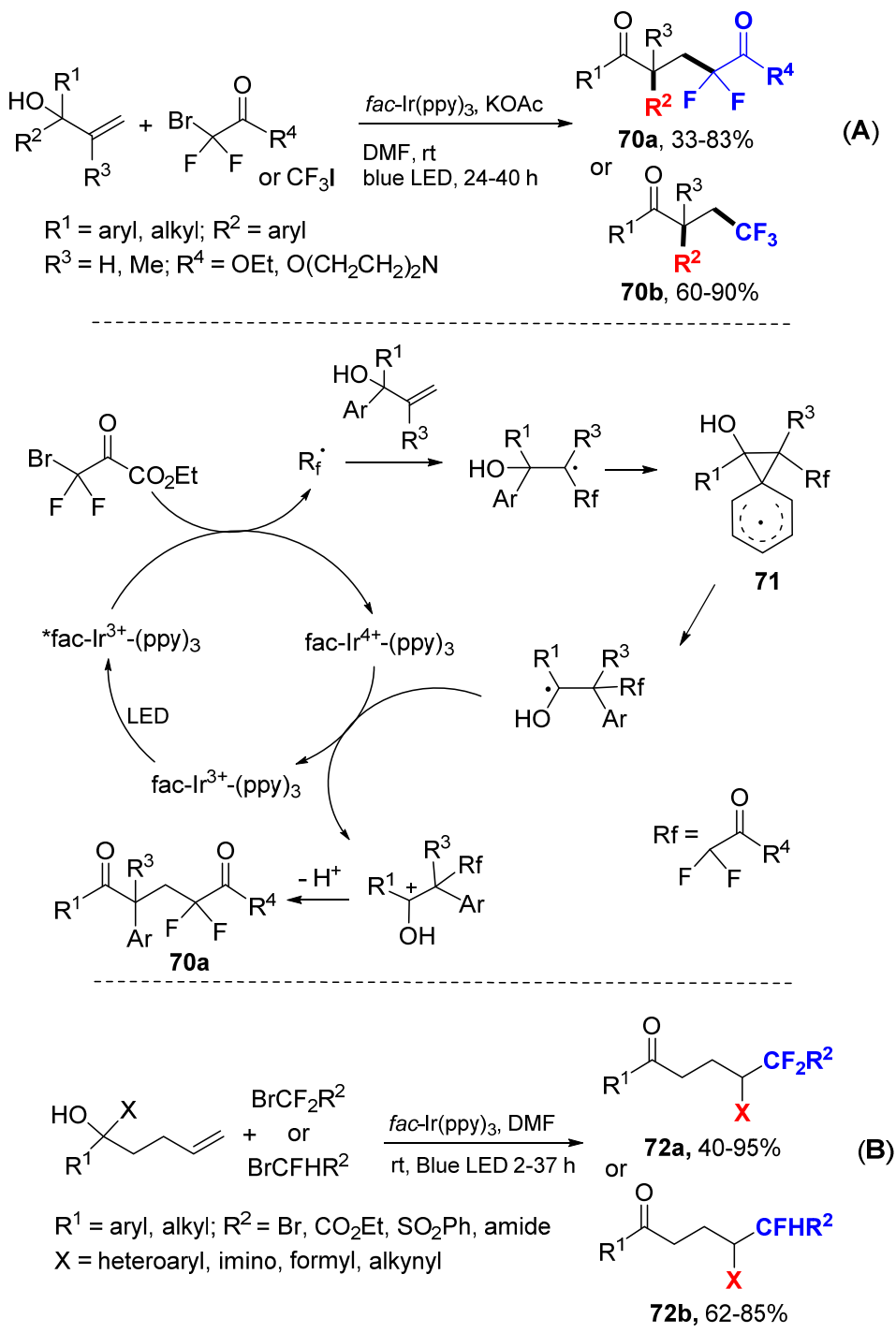

Scheme 45. Difunctionalization involving a 1,2- or 1,4-group migration. (A) 1,2-migration reaction. (B) 1,4-migration reaction. * excited catalyst.

The Ma and Li group reported a photoredox-catalyzed 1,6-oxyfluoroalkylation of alkenes using DMSO as an oxidant and also a solvent (Scheme 46) [78]. The perfluoroalkyl $\left(\mathrm{R}_{\mathrm{f}}\right)$ radicals are produced from $\mathrm{R}_{\mathrm{f}} \mathrm{Br}$ through the photoredox of the Ir-catalyst in the assistance of the Ag salt. The benzylic radicals generated from the addition of $R_{\mathrm{f}}$ radicals to alkenes undergo 1,5-hydrogen atom transfer (1,5-HAT) and then the Kornblum reaction with DMSO to afford 1,6-oxyfluoroalkylated ketones 73. 


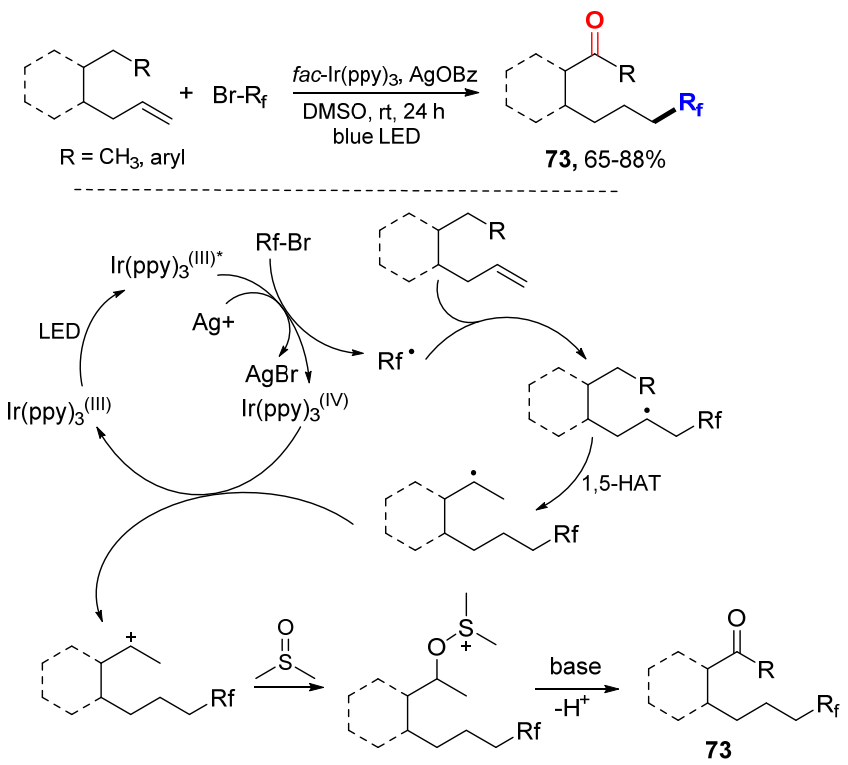

Scheme 46. 1,6-Oxyfluoroalkylation of alkenes. * excited catalyst.

The Zhu group developed a method to generate $\mathrm{CF}_{3}$ radical from $\mathrm{CF}_{3} \mathrm{SO}_{2} \mathrm{Na}$ and $\mathrm{PhI}\left(\mathrm{O}_{2} \mathrm{CCF}_{3}\right)_{2}$ for difunctionalization of unactivated alkenes involving distal heteroaryl group migration to form products 74 (Scheme 47A) [79]. The Umemoto's reagent is employed as $\mathrm{CF}_{3}$ radical source for trifluoromethylative alkynylation of unactivated alkenes to form products 75 through photocatalyzed reaction involving the alkynyl migration reaction (Scheme 47B) [80]. The Wang group reported an electrochemical reaction for fluoroalkylarylation of unactivated alkenes to form products $\mathbf{7 6}$ using $\mathrm{R}_{\mathrm{f}} \mathrm{SO}_{2} \mathrm{Na}$ as fluorocarbon radical source (Scheme 47C) [81]. All these three reactions involve distal group migration and their mechanisms are similar to that presented in Scheme 14.
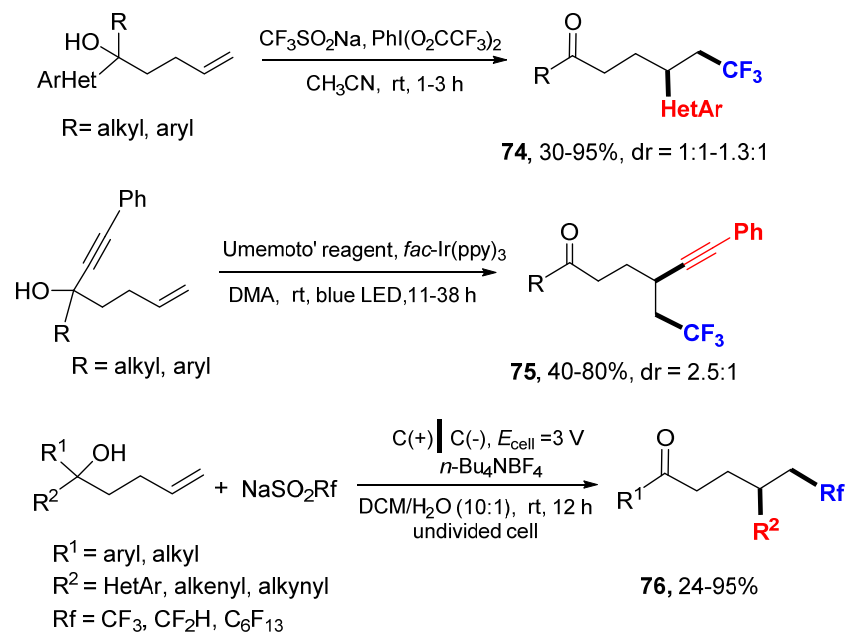

Scheme 47. Fluorocarbon radical reactions involving distal group migration. (A) heteroaryl group migration reaction. (B) alkynyl group migration reaction. (C) electrochemical reaction conditions.

The Studer group reported a method for perfluoroalkyltriflation of alkynes using phenyl(perfluoroalkyl)iodonium triflates 78 in the presence of $\mathrm{CuCl}$ to afford selective E-difunctionalized alkene products 77 (Scheme 48) [82]. $R_{\mathrm{f}}$ radicals generated from 78 through reductive SET with $\mathrm{CuCl}$ add to alkynes to from vinyl radicals and then vinylic cations after oxidation. Trapping of cations with triflate anion affords the products 77 . 


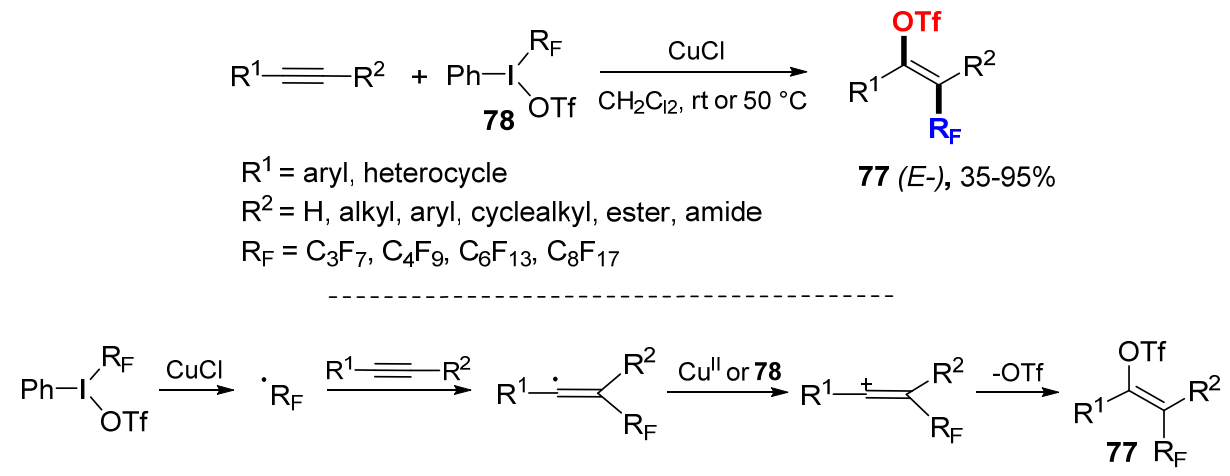

Scheme 48. Perfluoroalkyltriflation of alkynes.

Han and coworkers reported photoredox catalytic ketofluoromethylation of alkynes (Scheme 49) [83]. The $\mathrm{CF}_{3}$ radical generated from Umemoto's reagent through reductive SET add to alkynes to produce vinyl radicals which are oxidized to vinyl cations by SET and then trapped by $\mathrm{H}_{2} \mathrm{O}$ to give products 79 after deprotonation with $\mathrm{BF}_{4}{ }^{-}$and enol-keto tautomerization.<smiles>[R]C#Cc1ccc([R])cc1</smiles>

$\mathrm{R}^{1}=\mathrm{H}$, amide, alkyl, halogen, alkoxy $\mathrm{R}^{2}=\mathrm{H}, \mathrm{CF}_{3}$, alkyl, ketone

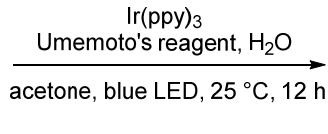

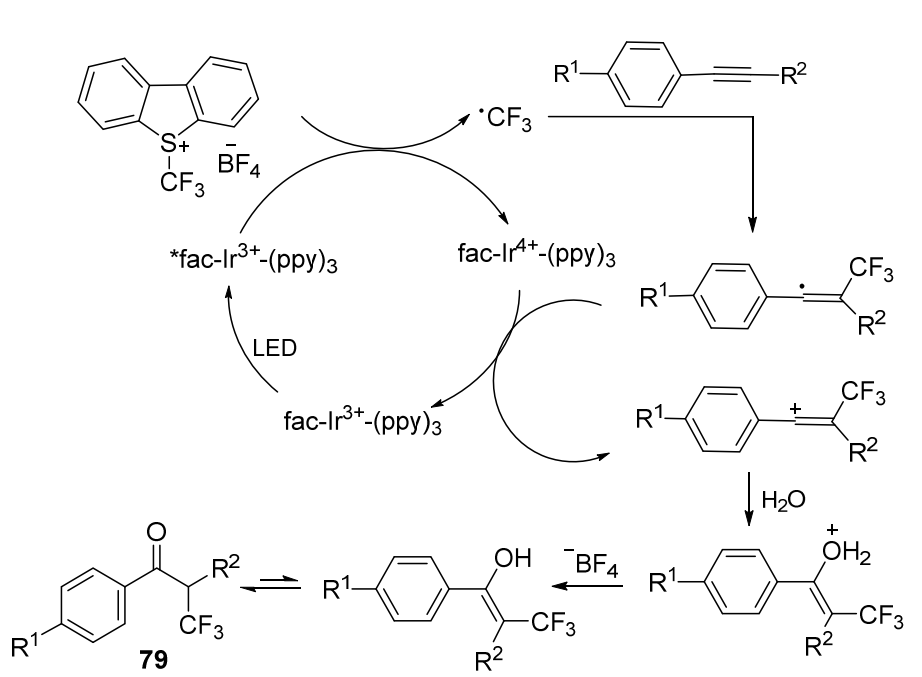

Scheme 49. Ketotrifluoromethylation of alkynes. * excited catalyst.

The Zhu and Li group reported photoredox-catalyzed remote ketofluoroalkylation and hydroxytrifluoromethylation of alkynes using $\mathrm{R}_{\mathrm{f}} \mathrm{X}$ or Umemoto's reagent as fluoroalkyl source and DMSO or $\mathrm{H}_{2} \mathrm{O}$ as oxygen source for the synthesis of $\varepsilon$-oxygenated fluoroalkylated (Z)-alkenes $\mathbf{8 0}$ and $\mathbf{8 1}$ (Scheme 50) [84]. In the reaction with the Umemoto's reagent, the $\mathrm{CF}_{3}$ radical generated through SET adds to alkynes to form vinyl radicals which then undergo 1,5-HAT followed by oxidative SET to form cationic intermediates $\mathbf{8 2}$ with a (Z)-alkenyl group. Nucleophilic attack of 82 by DMSO followed by the Kornblum oxidation produces trifluoromethyl (Z)-alkenyl ketones 80. Alternatively, using $\mathrm{H}_{2} \mathrm{O}$ instead of DMSO as a nucleophile, trifluoromethylated (Z)-alkenols $\mathbf{8 1}$ are produced from the reaction. 


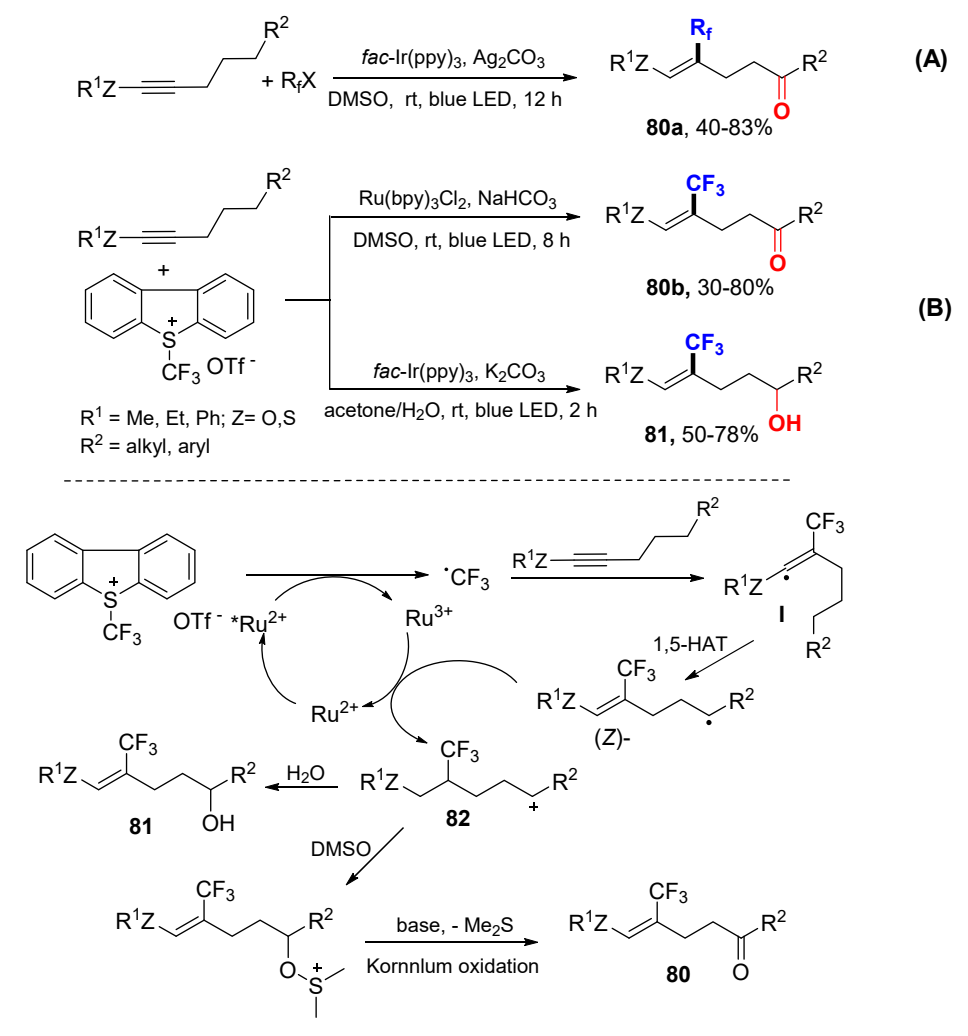

Scheme 50. Photoredox-catalyzed remote oxyfluoroalkylation. (A) $\mathrm{R}_{\mathrm{f}}$-involved reaction. (B) $\mathrm{CF}_{3^{-}}$ involved reaction. * excited catalyst.

\section{Other Radical-Initiated Reactions}

Other than above mentioned difunctionalization reactions initiated with $\mathrm{C}_{-}, \mathrm{N}_{-}, \mathrm{O}-$, $\mathrm{P}-$, and fluorocarbon-radicals, we found a single example of iodo radical-initiated difunctionalization reported by the Zhu group (Scheme 51) [85]. The reaction of TBHP with $\mathrm{I}_{2}$ generates $t$-BuOI and HOI. Radical iodination of styrenes followed by oxidation to cations and nucleophilic attacking by TBHP give iodoalkylperoxylated products 83 .

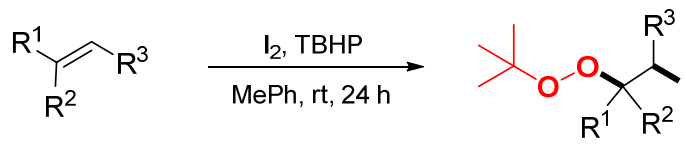

$\mathrm{R}^{1}=$ alkyl, aryl; $\mathrm{R}^{2}=$ alkyl

$83,70-85 \%$

$\mathrm{R}^{3}=\mathrm{H}$, alkyl

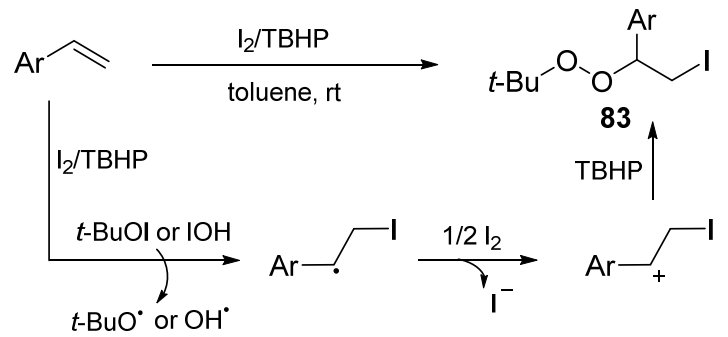

Scheme 51. Iodoalkylperoxylation of alkenes.

\section{Conclusions}

This article summarizes the radical and nucleophilic difunctionalization reactions of alkenes and alkynes involving a cascade process of radical generation, radical addition, oxidation to cation, and trapping by a nucleophile. This one-pot reaction process demonstrates 
the step and pot economies as well as operational simplicity. A wide range $\mathrm{C}-_{-}, \mathrm{O}-, \mathrm{N}-, \mathrm{S}-$, $\mathrm{P}$-centered and fluorocarbon radicals are used in combination with $\mathrm{C}-, \mathrm{N}-, \mathrm{O}-$-centered and halogenic nucleophiles for difunctionalizations to show substrate versatility.

Currently active research techniques such as photoredox catalysis and electrochemical reactions have been applied to the difunctionalization reactions. It is worth noting that in addition to radical and nucleophilic difunctionalization reactions presented in this paper, there are other radical-initiated difunctionalization reactions such as radical chain reactions, radical-radical coupling with or without metal catalysis. In some reaction processes, the radical difunctionalizations could have more than one pathways. The complex reaction processes have attracted the attention of synthetic chemists to conduct mechanism studies which could lead to the discovery of new reactions. We have no doubt that radical additioninitiated difunctionalization reactions for making molecules with potential biological, medical and functional material utilities will become important tools in organic synthesis.

Author Contributions: H.Y. literature search, review and original manuscript writing. W.H. revision of text and chem-drawing. W.Z. revision and supervision. All authors have read and agreed to the published version of the manuscript.

Funding: This research received no external funding.

Data Availability Statement: Data sharing not applicable.

Conflicts of Interest: The authors declare no conflict of interests.

\section{References}

1. Chatgilialoglu, C.; Studer, A. (Eds.) Encyclopedia of Radicals in Chemistry, Biology and Materials; Wiley: Chichester, UK, 2012.

2. Zard, S.Z. Radical Reactions in Organic Synthesis; Oxford University Press: Oxford, UK, 2003.

3. Yi, H.; Zhang, G.T.; Wang, H.M.; Huang, Z.Y.; Wang, J.; Singh, A.K.; Le, A.W. Radical-mediated remote functional group migration. Chem. Rev. 2017, 117, 9016-9085. [CrossRef]

4. Wu, X.X.; Zhu, C. Radical-mediated remote functional group migration. Acc. Chem. Res. 2020, 53, 1620-1636. [CrossRef]

5. Li, W.P.; Xu, W.T.; Xie, J.; Yu, S.Y.; Zhu, C.J. Distal radical migration strategy: An emerging synthetic means. Chem. Soc. Rev. 2018, 47, 654-667. [CrossRef]

6. McCarroll, A.J.; Walton, J.C. Programming organic molecules: Design and management of organic syntheses through free-radical cascade processes. Angew. Chem. Int. Ed. 2001, 40, 2224-2248. [CrossRef]

7. Prier, C.K.; Rankic, D.A.; MacMillan, D.W.C. Visible light photoredox catalysis with transition metal complexes: Applications in organic Synthesis. Chem. Rev. 2013, 113, 5322-5363. [CrossRef]

8. Yan, M.; Kawamata, Y.; Baran, P.S. Synthetic organic electrochemical methods since 2000: On the verge of a renaissance. Chem. Rev. 2017, 117, 13230-13319. [CrossRef]

9. Siu, J.C.; Fu, N.K.; Lin, S. Catalyzing electrosynthesis: A homogeneous electrocatalytic approach to reaction discovery. Acc. Chem. Res. 2020, 53, 547-560. [CrossRef]

10. Lan, X.-W.; Wang, N.-X.; Xing, Y. Recent advances in radical difunctionalization of simple alkenes. Eur. J. Org. Chem. 2017, 2017, 5821-5851. [CrossRef]

11. Li, Z.L.; Fang, G.C.; Gu, Q.S.; Liu, X.Y. Recent advances in copper-catalysed radical-involved asymmetric 1,2-difunctionalization of alkenes. Chem. Soc. Rev. 2020, 49, 32-48. [CrossRef] [PubMed]

12. Bao, X.Z.; Li, J.; Jiang, W.; Huo, C.D. Radical-mediated difunctionalization of styrenes. Synthesis 2019, 51, 4507-4530. [CrossRef]

13. Jiang, H.; Studer, A. Intermolecular radical carboamination of alkenes. Chem. Soc. Rev. 2020, 49, 1790-1811. [CrossRef] [PubMed]

14. Gao, Y.; Tang, G.; Zhao, Y.F. Recent progress toward organophosphorus compounds based on phosphorus-centered radical difunctionalizations. Phosphorus Sulfur Silicon Relat. Elem. 2017, 192, 589-596. [CrossRef]

15. Lin, J.; Song, R.J.; Hu, M.; Li, J.H. Recent advances in the intermolecular oxidative difunctionalization of alkenes. Chem. Rec. 2019, 19, 440-451. [CrossRef] [PubMed]

16. Koike, T.; Akita, M. A versatile strategy for difunctionalization of carbon-carbon multiple bonds by photoredox catalysis. Org. Chem. Front. 2016, 3, 1345-1349. [CrossRef]

17. Besset, T.; Poisson, T.; Pannecoucke, X. Direct vicinal difunctionalization of alkynes: An efficient approach towards the synthesis of highly functionalized fluorinated alkenes. Eur. J. Org. Chem. 2015, 2015, 2765-2789. [CrossRef]

18. Dai, Q.; Jiang, Y.; Yu, J.T.; Cheng, J. Peroxide: A novel methylating reagent. Synthesis 2016, 48, 329-339. [CrossRef]

19. Reiser, O. Shining light on copper: Unique opportunities for visible-light-catalyzed atom transfer radical addition reactions and related processes. Acc. Chem. Res. 2016, 49, 1990-1996. [CrossRef]

20. Wang, F.; Chen, P.H.; Liu, G.S. Copper-catalyzed radical relay for asymmetric radical transformations. Acc. Chem. Res. 2018, 51, 2036-2046. [CrossRef] 
21. Xuan, J.; Studer, A. Radical cascade cyclization of 1,n-enynes and diynes for the synthesis of carbocycles and heterocycles. Chem. Soc. Rev. 2017, 46, 4329-4346. [CrossRef]

22. Wang, Y.; Zhang, L.; Yang, Y.H.; Zhang, P.; Du, Z.T.; Wang, C.Y. Alkene oxyalkylation enabled by merging rhenium catalysis with hypervalent Iodine(III) reagents via decarboxylation. J. Am. Chem. Soc. 2013, 135, 18048-18051. [CrossRef]

23. Ouyang, X.H.; Song, R.J.; Hu, M.; Yang, Y.; Li, J.H. Silver-mediated intermolecular 1,2-alkylarylation of styrenes with $\alpha$-carbonyl alkyl bromides and indoles. Angew. Chem. Int. Ed. 2016, 55, 3187-3191. [CrossRef] [PubMed]

24. Jian, W.J.; Ge, L.; Jiao, Y.H.; Qian, B.; Bao, H.L. Iron-catalyzed decarboxylative alkyl etherification of vinylarenes with aliphatic acids as the alkyl source. Angew. Chem. Int. Ed. 2017, 56, 3650-3654. [CrossRef] [PubMed]

25. Murata, Y.; Shimada, T.; Nishikata, T. Radical and cation crossover reaction system enables synthesis of complex aliphatic chains possessing functionalized quaternary carbons. Bull. Chem. Soc. Jpn. 2019, 92, 1419-1429. [CrossRef]

26. Li, W.Y.; Wang, Q.Q.; Yang, L. Fe-catalyzed radical-type difunctionalization of styrenes with aliphatic aldehydes and trimethylsilyl azide via a decarbonylative alkylation-azidation cascade. Org. Biomol. Chem. 2017, 15, 9987-9991. [CrossRef]

27. Bao, X.; Yokoe, T.; Ha, T.M.; Wang, Q.; Zhu, J.P. Copper-catalyzed methylative difunctionalization ofalkenes. Nat. Commun. 2018, 9, 3725. [CrossRef]

28. Liu, S.S.; Klussmann, M. Acid promoted radical-chain difunctionalization of styrenes with stabilized radicals and (N,O)nucleophiles. Chem. Commun. 2020, 56, 155-1560. [CrossRef]

29. Fumagalli, G.; Boyd, S.; Greaney, M.F. Oxyarylation and aminoarylation of styrenes using photoredox catalysis. Org. Lett. 2013, 15, 4399-4401. [CrossRef]

30. Tlahuext-Aca, A.; Garza-Sanchez, R.A.; Glorius, F. Multicomponent oxyalkylation of styrenes enabled by hydrogen-bond-assisted photoinduced electron transfer. Angew Chem. Int. Ed. Engl. 2017, 56, 3708-3711. [CrossRef]

31. Yong, X.; Han, Y.F.; Li, Y.; Song, R.J.; Li, J.H. Alkylarylation of styrenes via direct C( $\left.\mathrm{sp}^{3}\right)-\mathrm{Br} / \mathrm{C}\left(\mathrm{sp}^{2}\right)-\mathrm{H}$ functionalization mediated by photoredox and copper cooperative catalysis. Chem. Commun. 2018, 91, 12816-12819. [CrossRef]

32. Lux, M.; Klussmann, M. Additions of aldehyde-derived radicals and nucleophilic $N$-alkylindoles to styrenes by photoredox catalysis. Org. Lett. 2020, 22, 3697-3701. [CrossRef]

33. Huang, X.Y.; Bergsten, T.M.; Groves, J.T. Manganese-catalyzed late-stage aliphatic C-H azidation. J. Am. Chem. Soc. 2015, 137, 5300-5303. [CrossRef] [PubMed]

34. Wu, Z.; Ren, R.G.; Zhu, C. Combination of a cyano migration strategy and alkene difunctionalization: The elusive selective azidocyanation of unactivated olefins. Angew. Chem. Int. Ed. 2016, 55, 10821-10824. [CrossRef] [PubMed]

35. Wang, M.; Wu, Z.; Zhang, B.; Zhu, C. Azidoheteroarylation of unactivated olefins through distal heteroaryl migration. Org. Chem. Front. 2018, 5, 1896-1899. [CrossRef]

36. Cong, F.; Wei, Y.; Tang, P. Combining photoredox and silver catalysis for azidotrifluoromethoxylation of styrenes. Chem. Commun. 2018, 54, 4473-4476. [CrossRef] [PubMed]

37. Guo, W.; Wang, Q.; Zhu, J. Selective 1, 2-Aminoisothiocyanation of 1, 3-dienes under visible light photoredox catalysis. Angew. Chem. Int. Ed. Engl. 2020. [CrossRef]

38. Xue, Q.; Xie, J.; Xu, P.; Hu, K.; Cheng, Y.; Zhu, C. Metal-free, n-Bu 4 NI-catalyzed regioselective difunctionalization of unactivated alkenes. ACS Catal. 2013, 3, 1365-1368. [CrossRef]

39. Xia, X.-F.; Gu, Z.; Liu, W.; Wang, H.; Xia, Y.; Gao, H.; Liu, X.; Liang, Y.-M. Metal-free three-component oxyazidation of alkenes with trimethylsilyl azide and N-hydroxyphthalimide. J. Org. Chem. 2015, 80, 290-295. [CrossRef]

40. Barthelemy, A.L.; Tuccio, B.; Magnier, E.; Dagousset, G. Alkoxyl radicals generated under photoredox catalysis: A strategy for anti-Markovnikov alkoxylation reactions. Angew. Chem. Int. Ed. Engl. 2018, 57, 13790-13794. [CrossRef]

41. Pan, X.-Q.; Zou, J.-P.; Yi, W.-B.; Zhang, W. Recent advances in sulfur-and phosphorous-centered radical reactions for the formation of $\mathrm{s}-\mathrm{c}$ and $\mathrm{p}-\mathrm{c}$ bonds. Tetrahedron 2015, 40, 7481-7529. [CrossRef]

42. Zhang, P.-Z.; Zhang, L.; Li, J.-A.; Shoberu, A.; Zou, J.-P.; Zhang, W. Phosphinoyl radical initiated vicinal cyanophosphinoylation of alkenes. Org. Lett. 2017, 19, 5537-5540. [CrossRef]

43. Yang, B.; Hou, S.M.; Ding, S.Y.; Zhao, X.N.; Gao, Y.; Wang, X.; Yang, S.D. Cerium (IV)-Promoted Phosphinoylation-Nitratation of Alkenes. Adv. Synth. Catal. 2018, 360, 4470-4474. [CrossRef]

44. Wang, Y.; Wang, W.; Tang, R.; Liu, Z.; Tao, W.; Fang, Z. Iron(iii)-catalyzed radical $\alpha$, $\beta$-aminophosphinoylation of styrenes with diphenylphosphine oxides and anilines. Org. Biomol. Chem. 2018, 16, 7782-7786. [CrossRef] [PubMed]

45. Chen, D.; Wu, Z.; Yao, Y.; Zhu, C. Phosphinoyl-functionalization of unactivated alkenes through phosphinoyl radical-triggered distal functional group migration. Org. Chem. Front. 2018, 5, 2370-2374. [CrossRef]

46. Xiang, Y.; Kuang, Y.; Wu, J. Generation of $\beta$-halo vinylsulfones through a multicomponent reaction with insertion of sulfur dioxide. Chem. Eur. J. 2017, 23, 6996-6999. [CrossRef] [PubMed]

47. Zong, Y.; Lang, Y.; Yang, M.; Li, X.; Fan, X.; Wu, J. Synthesis of $\beta$-sulfonyl amides through a multicomponent reaction with the insertion of sulfur dioxide under visible light irradiation. Org. Lett. 2019, 21, 1935-1938. [CrossRef] [PubMed]

48. Samanta, S.; Chatterjee, R.; Santra, S.; Hajra, A.; Khalymbadzha, I.A.; Zyryanov, G.V.; Majee, A. Mild, efficient, and metal-free radical 1,2-dithiocyanation of alkynes and alkenes at room temperature. ACS Omega 2018, 3, 13081-13088. [CrossRef]

49. Wang, Y.; Deng, L.; Mei, H.; Du, B.; Han, J.; Pan, Y. Electrochemical oxidative radical oxysulfuration of styrene derivatives with thiols and nucleophilic oxygen sources. Green Chem. 2018, 20, 3444-3449. [CrossRef] 
50. Zheng, M.-W.; Yuan, X.; Cui, Y.-S.; Qiu, J.-K.; Li, G.; Guo, K. Electrochemical sulfonylation/heteroarylation of alkenes via distal heteroaryl ipso-migration. Org. Lett. 2018, 20, 7784-7789. [CrossRef]

51. Yuan, Y.; Cao, Y.; Lin, Y.; Li, Y.; Huang, Z.; Lei, A. Electrochemical oxidative alkoxysulfonylation of alkenes using sulfonyl hydrazines and alcohols with hydrogen evolution. ACS Catal. 2018, 8, 10871-10875. [CrossRef]

52. Yuan, Y.; Chen, Y.; Tang, S.; Huang, Z.; Lei, A. Electrochemical oxidative oxysulfenylation and aminosulfenylation of alkenes with hydrogen evolution. Sci. Adv. 2018, 4, eaat5312. [CrossRef]

53. Chakrasali, P.; Kim, K.; Jung, Y.-S.; Kim, H.; Han, S.B. Visible-light-mediated photoredox-catalyzed regio-and stereoselective chlorosulfonylation of alkynes. Org. Lett. 2018, 20, 7509-7513. [CrossRef] [PubMed]

54. Ni, B.; Zhang, B.; Han, J.; Peng, B.; Shan, Y.; Niu, T. Heterogeneous carbon nitrides photocatalysis multicomponent hydrosulfonylation of alkynes to access $\beta$-keto sulfones with the insertion of sulfur dioxide in aerobic aqueous medium. Org. Lett. 2020, 22, 670-674. [CrossRef] [PubMed]

55. Tang, N.; Yang, S.; Wu, X.; Zhu, C. Visible-light-induced carbosulfonylation of unactivated alkenes via remote heteroaryl and oximino migration. Tetrahedron 2019, 75, 1639-1646. [CrossRef]

56. Wang, J.; Sánchez-Roselló, M.; Aceña, J.L.; del Pozo, C.; Sorochinsky, A.E.; Fustero, S.; Soloshonok, V.A.; Liu, H. Fluorine in pharmaceutical industry: Fluorine-containing drugs introduced to the market in the last decade (2001-2011). Chem. Rev. 2014, 114, 2432-2506. [CrossRef]

57. Gouverneur, V.; Seppelt, K. Introduction: Fluorine chemistry. Chem. Rev. 2015, 115, 563-565. [CrossRef]

58. Barata-Vallejo, S.; Postigo, A. Metal-mediated radical perfluoroalkylation of organic compounds. Coord. Chem. Rev. 2013, 257, 3051-3069. [CrossRef]

59. Charpentier, J.; Fruh, N.; Togni, A. Electrophilic trifluoromethylation by use of hypervalent iodine reagents. Chem. Rev. 2015, 115, 650-682. [CrossRef]

60. Zhang, C. Recent advances in trifluoromethylation of organic compounds using Umemoto's reagents. Org. Biomol. Chem. 2014, 12, 6580-6589. [CrossRef]

61. Yasu, Y.; Koike, T.; Akita, M. Three-component oxytrifluoromethylation of alkenes: Highly efficient and regioselective difunctionalization of $\mathrm{C}=\mathrm{C}$ bonds mediated by photoredox catalysts. Angew. Chem. Int. Ed. Engl. 2012, 51, 9567-9571. [CrossRef]

62. Yasu, Y.; Koike, T.; Akita, M. Intermolecular aminotrifluoromethylation of alkenes by visible-light-driven photoredox catalysis. Org. Lett. 2013, 15, 2136-2139. [CrossRef]

63. Dagousset, G.; Carboni, A.; Magnier, E.; Masson, G. Photoredox-induced three-component azido- and aminotrifluoromethylation of alkenes. Org. Lett. 2014, 16, 4340-4343. [CrossRef]

64. Zhou, X.; Li, G.; Shao, Z.; Fang, K.; Gao, H.; Li, Y.; She, Y. Four-component acyloxy-trifluoromethylation of arylalkenes mediated by a photoredox catalyst. Org. Biomol. Chem. 2019, 17, 24-29. [CrossRef]

65. Tomita, R.; Yasu, Y.; Koike, T.; Akita, M. Combining photoredox-catalyzed trifluoromethylation and oxidation with DMSO: Facile synthesis of $\alpha$-trifluoromethylated ketones from aromatic alkenes. Angew. Chem. 2014, 126, 7272-7276. [CrossRef]

66. Carboni, A.; Dagousset, G.; Magnier, E.; Masson, G. Photoredox-induced three-component oxy-, amino-, and carbotrifluoromethylation of enecarbamates. Org. Lett. 2014, 16, 1240-1243. [CrossRef] [PubMed]

67. Zhang, L.; Zhang, G.; Wang, P.; Li, Y.; Lei, A. Electrochemical oxidation with Lewis-acid catalysis leads to trifluoromethylative difunctionalization of alkenes using $\mathrm{CF}_{3} \mathrm{SO}_{2} \mathrm{Na}$. Org. Lett. 2018, 20, 7396-7399. [CrossRef] [PubMed]

68. Oh, S.H.; Malpani, Y.R.; Ha, N.; Jung, Y.-S.; Han, S.B. Vicinal difunctionalization of alkenes: Chlorotrifluoromethylation with $\mathrm{CF}_{3} \mathrm{SO}_{2} \mathrm{Cl}$ by photoredox catalysis. Org. Lett. 2014, 16, 1310-1313. [CrossRef]

69. Han, H.S.; Lee, Y.J.; Jung, Y.-S.; Han, S.B. Stereoselective photoredox-catalyzed chlorotrifluoromethylation of alkynes: Synthesis of tetrasubstituted alkenes. Org. Lett. 2017, 19, 1962-1965. [CrossRef]

70. Duan, Y.; Li, W.; Xu, P.; Zhang, M.; Cheng, Y.; Zhu, C. Visible-light-induced three-component 1, 2-difluoroalkylarylation of styrenes with $\alpha$-carbonyl difluoroalkyl bromides and indoles. Org. Chem. Front. 2016, 3, 1443-1446. [CrossRef]

71. Yang, Q.; Li, C.; Qi, Z.C.; Qiang, X.Y.; Yang, S.D. Photocatalyzed intermolecular aminodifluoromethylphosphonation of alkenes: Facile synthesis of $\alpha, \alpha$-difluoro- $\gamma$-aminophosphonates. Chem. Eur. J. 2018, 24, 14363-14367. [CrossRef]

72. Arai, Y.; Tomita, R.; Ando, G.; Koike, T.; Akita, M. Oxydifluoromethylation of alkenes by photoredox catalysis: Simple synthesis of $\mathrm{CF}_{2} \mathrm{H}$-containing alcohols. Chem. Eur. J. 2016, 22, 1262-1265. [CrossRef]

73. Lin, Q.-Y.; Ran, Y.; Xu, X.-H.; Qing, F.-L. Photoredox-catalyzed bromodifluoromethylation of alkenes with (difluoromethyl) triphenylphosphonium bromide. Org. Lett. 2016, 18, 2419-2422. [CrossRef]

74. Ran, Y.; Lin, Q.-Y.; Xu, X.-H.; Qing, F.-L. Visible light induced oxydifluoromethylation of styrenes with difluoromethyltriphenylphosphonium bromide. J. Org. Chem. 2016, 81, 7001-7007. [CrossRef] [PubMed]

75. Daniel, M.; Dagousset, G.; Diter, P.; Klein, P.A.; Tuccio, B.; Goncalves, A.M.; Masson, G.; Magnier, E. Fluorinated sulfilimino iminiums: Efficient and versatile sources of perfluoroalkyl radicals under photoredox catalysis. Angew. Chem. 2017, 129, 4055-4059. [CrossRef]

76. Xu, P.; Hu, K.; Gu, Z.; Cheng, Y.; Zhu, C. Visible light promoted carbodifluoroalkylation of allylic alcohols via concomitant 1 , 2-aryl migration. Chem. Commun. 2015, 51, 7222-7225. [CrossRef]

77. Yu, J.; Wang, D.; Xu, Y.; Wu, Z.; Zhu, C. Distal functional group migration for visible-light induced carbo-difluoroalkylation/monofluoroalkylation of unactivated alkenes. Adv. Synth. Catal. 2018, 360, 744-750. [CrossRef] 
78. Li, L.; Luo, H.; Zhao, Z.; Li, Y.; Zhou, Q.; Xu, J.; Li, J.; Ma, Y.-N. Photoredox-catalyzed remote difunctionalizations of alkenes To synthesize fluoroalkyl ketones with dimethyl sulfoxide as the oxidant. Org. Lett. 2019, 21, 9228-9231. [CrossRef] [PubMed]

79. Wu, Z.; Wang, D.; Liu, Y.; Huan, L.; Zhu, C. Chemo- and regioselective distal heteroaryl ipso-migration: A general protocol for heteroarylation of unactivated alkenes. J. Am. Chem. Soc. 2017, 139, 1388-1391. [CrossRef] [PubMed]

80. Xu, Y.; Wu, Z.; Jiang, J.; Ke, Z.; Zhu, C. Merging distal alkynyl migration and photoredox catalysis for radical trifluoromethylative alkynylation of unactivated olefins. Angew. Chem. 2017, 129, 4616-4619. [CrossRef]

81. Zou, Z.; Zhang, W.; Wang, Y.; Kong, L.; Karotsis, G.; Wang, Y.; Pan, Y. Electrochemically promoted fluoroalkylation-distal functionalization of unactivated alkenes. Org. Lett. 2019, 21, 1857-1862. [CrossRef] [PubMed]

82. Wang, X.; Studer, A. Regio- and stereoselective radical perfluoroalkyltriflation of alkynes using phenyl (perfluoroalkyl) iodonium triflates. Org. Lett. 2017, 19, 2977-2980. [CrossRef]

83. Malpani, Y.R.; Biswas, B.K.; Han, H.S.; Jung, Y.-S.; Han, S.B. Multicomponent oxidative trifluoromethylation of alkynes with photoredox catalysis: Synthesis of $\alpha$-trifluoromethyl ketones. Org. Lett. 2018, 20, 1693-1697. [CrossRef] [PubMed]

84. Shang, T.; Zhang, J.; Zhang, Y.; Zhang, F.; Li, X.-S.; Zhu, G. Photocatalytic remote oxyfluoroalkylation of heteroalkynes: Regio-, stereo-, and site-selective access to complex fluoroalkylated (Z)-alkenes. Org. Lett. 2020, 22, 3667-3672. [CrossRef] [PubMed]

85. Wang, H.; Chen, C.; Liu, W.; Zhu, Z. Difunctionalization of alkenes with iodine and tert-butyl hydroperoxide (TBHP) at room temperature for the synthesis of 1-(tert-butylperoxy)-2-iodoethanes. Beilstein J. Org. Chem. 2017, 13, 2023-2027. [CrossRef] [PubMed] 